\title{
ON THE EXCEPTIONAL SET IN GOLDBACH'S PROBLEM
}

\author{
By
}

Hiroshi MikawA

\section{Introduction.}

Let $E(x)$ denote the number of even integers not exceeding $x$ that are not representable as a sum of two primes. The Goldbach conjecture asserts $E(x)$ $\ll 1$. Unfortunately this is far from our reach. In 1923 G. H. Hardy and J.E. Littlewood [4] showed, on assuming the extended Riemann hypothesis, that

$$
E(x) \ll x^{1 / 2+\varepsilon}
$$

for any $\varepsilon>0$. After the fundamental work of I. M. Vinogradov [18], several authors have unconditionally given the non-trivial bounds for $E(x)$. The best one of these is due to H. L. Montgomery and R. C. Vaughan. In 1975 they [13] showed that there exists a positive constant $\delta$ such that

$$
E(x) \ll x^{1-\delta} .
$$

Chen J.-r. [2] gave an explicit value of $\delta$, which is very small.

In $1973 \mathrm{~K}$. Ramachandra [16] proved that, for any $A>0$,

$$
E\left(x+x^{\theta}\right)-E(x) \ll x^{\theta}(\log x)^{-A}
$$

providing

$$
\frac{7}{12}<\theta \leqq 1
$$

This bound $7 / 12$ comes from a zero density estimate for the Dirichlet $L$-series. In 1981 Lou S.-t. and Yao Q. [9] attempted to sharpen the inequality (2). Later Yao [20] replaced, in the same range of $\theta$ as (3), the right hand side of (2) by $x^{\theta(1-\delta)}$ with some $\delta>0$.

It is of some interest, from the point of veiw of (1), to demonstrate the formula (2) for $\theta$ less than $1 / 2$. We shall present such a result.

Theorem. Let $A>0$ and $7 / 48<\theta \leqq 1$ be given. Then we have

$$
E\left(x+x^{\theta}\right)-E(x) \ll x^{\theta}(\log x)^{-A}
$$

Received July 26, 1991, Revised March 9, 1992. 
where the implied constant depends only on $A$ and $\theta$.

This work is inspired by the article of D. Wolke [19]. On combining the argument of [16] with the result of [19], one may prove our assertion for $\theta>(7 / 12) \cdot(5 / 8)$, which is less than $1 / 2$ surely. We appeal to the device of $\mathrm{H}$. Iwaniec and M. Jutila [7]. See also [14]. Using the method in our previous papers $[10,11]$, we then conclude the bound $\theta>(7 / 12) \cdot(1 / 4)$.

Our notation is standard or self-explanatory. For a real number $t$, we write $\phi(x)=[x]-x+1 / 2, e(x)=e^{2 \pi i x}$ and $\|x\|=\min _{n \in \mathbf{Z}}|x-n|$. The convention $n \sim N$ means that $N<n \leqq N^{\prime} \leqq 2 N . \quad \rho$ in $\sum_{\rho(x)}$ runs through the set of non-trivial zeros of $L(s, \chi) . \quad c_{i}$ 's denote certain positive absolute constants. For simplicity, we write $\mathcal{L}=\log x . \quad F$ in $\mathcal{L}^{F}$ stands for a positive numerical constant, which is not the same at each occurrence.

I would like to thank Professor S. Uchiyama for suggestion and encouragement. I would also like to thank the referee for careful reading of this long paper.

\section{Lemmas.}

LeMmA 1. Let $1 \leqq a \leqq q,(a, q)=1$. The Hurwitz zeta-function $\zeta(s, a / q)$ is regular, except for a simple pole at $s=1$, of residue 1 . Also, it satisfies the growth conditions:

$$
\zeta\left(s, \frac{a}{q}\right) \ll\left\{\begin{array}{cl}
q+\exp \left(c_{1}(\log \log x)^{3}\right) ; 1-c_{2}(\log x)^{-4 / 5}< & \operatorname{Re}(s) \leqq 1+(\log x)^{-1} \\
& |\operatorname{Im}(s)| \leqq x \\
q^{1 / 2}+x^{1 / 6}(\log x) ; & |s-1| \geqq(\log x)^{-1} \\
& \mid \operatorname{Im}(s)=1 / 2 \\
q x^{(1-\operatorname{Re}(s)) / 3}(\log x) ; & 1 / 2 \leqq \operatorname{Re}(s) \leqq 1+(\log x)^{-1} \\
c_{3} \leqq & \operatorname{Im}(s) \mid \leqq x
\end{array}\right.
$$

Lemma 2. Let $N(\sigma, T, \chi)$ denote the number of zeros of $L(s, \chi)$ in the rectangle; $\operatorname{Re}(s) \geqq \sigma,|\operatorname{Im}(s)| \leqq T$. Let $A>0$ be given. If $q \leqq(\log T)^{A}$ and $\sigma \geqq$ $1-c_{4}(\log T)^{-4 / 5}$ then

$$
\sum_{x(\bmod q)} N(\sigma, T, \chi)=0 .
$$

For Lemmas 1 and 2, see [15, Kap. VIII Satz 5.3. Kap. IV Satz 5.3. Kap. IX Satz 4.2. Anhang Satz 9.1. Kap. VIII Satz 6.2.]. The following Lemma 3 is due to H. L. Montgomety [12, Theorem 12.1] and M. N. Huxley [5]. Lemma 
4 follows from [12, Theorem 7.6] immediately.

LEMmA 3 . Let $1 / 2 \leqq \sigma \leqq 1$. For any $\varepsilon>0$,

$$
\sum_{\chi(\bmod q)} N(\sigma, T, \chi) \ll(q T)^{(12 / 5+\varepsilon)(1-\sigma)}(\log q T)^{14}
$$

where the implied constant depends only on $\varepsilon$.

LEMMA 4. For $k=1,2$,

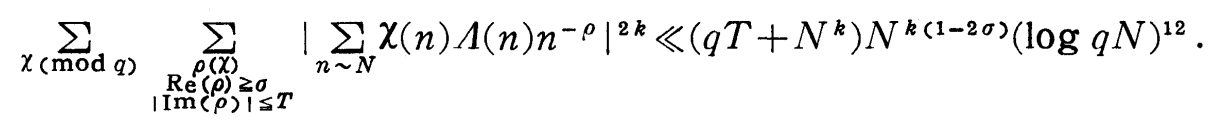

Let $\Omega$ be a non-negative arithmetical function with a compact support, and $\mathscr{P}$ a set of primes. For $z>2$, put

$$
S(\Omega, \mathscr{P}, z)=\sum_{(a, P(z))=1} \Omega(a)
$$

where

$$
P(z)=\prod_{\substack{p<z \\ p \in \mathcal{P}}} p
$$

Suppose that, for a square-free $d$ composed by primes $\in \mathscr{Q}$,

$$
\sum_{a \equiv 0(\bmod d)} \Omega(a)=\frac{\omega(d)}{d} X+r(\Omega, d)
$$

where $X$ is an approximation of $\Sigma \Omega(a)$ and $\omega(d)$ is a multiplicative function which satisfies some conditions of regularity. Write

$$
V(z)=\prod_{\substack{p<z \\ p \in \mathcal{P}}}\left(1-\frac{\omega(p)}{p}\right) \text {. }
$$

LEMma 5. Let $z, D>2$ and $s=\log D / \log z$. For $2 \leqq s \leqq 4$ we have

$$
S(\Omega, \mathscr{P}, z) \geqq V(z) X\left\{\frac{2 e^{r} \log (s-1)}{s}+O\left((\log \log D)^{-1}\right)\right\}+R,
$$

where $\gamma$ is the Euler constant and the remainder term $R$ has the form

$$
R=\sum_{d \mid P(z)} \lambda_{d}(D, z) r(\Omega, d) .
$$

Here, the weights $\left(\lambda_{d}\right)=\left(\lambda_{d}(D, z)\right)$ satisfy that

$$
\begin{aligned}
& \lambda_{d}=0 \quad \text { if } \quad d \geqq D, \\
& \left|\lambda_{d}\right| \leqq \mu^{2}(d) .
\end{aligned}
$$

Moreover, on writing $z_{i}=\exp \left((\log D)(\log \log D)^{-i / 10}\right)$, if $\lambda_{d} \neq 0$ for $d>z_{1} z$ then $\lambda_{d}$ is decomposed into the shape 


$$
\lambda_{d}=\sum_{h \leqq \log } \sum_{d=m p} \sum_{P_{h} \leq p_{<P_{h^{\prime}}}} \alpha(h, m)
$$

where

$$
z_{2} \leqq P_{h}<D^{1 / 4}, \quad P_{h}^{\prime}=P_{h} z_{9} \quad \text { and } \quad|\alpha(h, m)| \leqq 1 .
$$

This is the Rosser-Iwaniec linear sieve $[6,14]$. We explain the decomposition (2.1). It follows from $[6,14]$ that if $\lambda_{d} \neq 0$ then $d$ is the form

$$
d=\nu \text { or } \nu p_{1} \cdots p_{r}
$$

Here,

$$
\begin{aligned}
& \nu \leqq z_{1} \quad D_{i} \leqq p_{i}<D_{i} z_{9} \\
& \nu\left|P\left(z_{2}\right) \quad p_{i}\right| P(z) / P\left(z_{2}\right),
\end{aligned}
$$

$\left(D_{1}, \cdots, D_{r}\right)$ is a subsequence of $\left(z_{2} z_{9}{ }^{n}\right)_{n \geqq 0}$ such that

$$
\begin{gathered}
D_{1} \geqq \cdots \geqq D_{r}, \\
D_{1} \cdots D_{2 l-1} D_{2 l}{ }^{3}<D \quad \text { for all } 1 \leqq l \leqq r / 2 .
\end{gathered}
$$

Moreover, the coefficient of $d$ depends only on $\nu$ and $\left(D_{1}, \cdots, D_{r}\right)$. And, the number of $\left(D_{1}, \cdots, D_{r}\right)$ 's is at most $\log D$.

Now, if $d>z_{1} z$ then $z_{1} z<d=\nu p_{1} \cdots p_{r} \leqq z_{1} z^{r}$, whence $r \geqq 2$. Thus, $D_{2}^{4} \leqq$ $D_{1} D_{2}{ }^{3}<D$. Since $D_{i} \geqq z_{2}$, we obtain (2.1).

Next lemma is the combinatrial identity of R.C. Vaughan [17].

LEMMA 6. If $Y<l \leqq X Y$ then

$$
\Lambda(l)=\sum_{\substack{l=m n \\ n>Y}}\left(\sum_{\substack{d \backslash m \\ d \leq X}} \mu(d)\right) \Lambda(n)
$$

LEMMA 7. Let $1<\Delta<x / 2$. For arbitrary complex numbers $a_{n}$, put

$$
\mathcal{F}=\left(\frac{\Delta}{2}\right)^{2} \int_{-1 / 2 \Delta}^{1 / 2 \Delta}\left|\sum_{x<n \leqslant 2 x} a_{n} e(\beta n)\right|^{2} d \beta \text {. }
$$

Then we have

$$
\mathscr{F} \ll \int_{x}^{2 x}\left|\sum_{t<n \leqq t+\Delta} a_{n}\right|^{2} d t+\Delta^{3}\left(\sup _{x<n \leq 2 x}\left|a_{n}\right|\right)^{2}
$$

LEMMA 8. Under the assumption of Lemma 7, we have

$$
\begin{aligned}
\mathcal{F} & \leqq \Delta \sum_{x<n \leqq 2 x}\left|a_{n}\right|^{2}+2 \operatorname{Re} \sum_{0<r \leqq \Delta}(\Delta-r) \sum_{\substack{x<m \\
m-n=r}} \sum_{\substack{n \leq 2 x \\
m=n}} a_{m} \bar{a}_{n} \\
& \ll \Delta \sum_{x<n \leqq 2 x}\left|a_{n}\right|^{2}+\Delta \sup _{t \leqq \Delta}\left|\sum_{0<r \leqq t} \sum_{x<n \leqq 2 x} \bar{a}_{n} a_{n+r}\right|+\Delta^{3}\left(\sup _{x<n \leqq 2 x}\left|a_{n}\right|\right)^{2} .
\end{aligned}
$$

Lemma 7 is [3, Lemma 1] and Lemma 8 follows from a familiar Fourier 
integral. We, however, reproduce the proof. It is well known that

$$
\max (0, \Delta-|x|)=\int_{-\infty}^{\infty} K(y, \Delta) e(x y) d y
$$

where

$$
K(y, \Delta)=\left(\frac{\sin \pi \Delta y}{\pi y}\right)^{2}
$$

Put

$$
\begin{aligned}
& \mathscr{D}=\sum_{x<m, n \leqq 2 x} a_{m} a_{n} \max (0, \Delta-|m-n|), \\
& \mathcal{S}=\int_{x}^{2 x}\left|\sum_{t<n \leqq t+\Delta} a_{n}\right|^{2} d t,
\end{aligned}
$$

and

$$
R=\Delta^{3}\left(\sup _{x<n \leqq 2 x}\left|a_{n}\right|\right)^{2}
$$

We shall show $\mathcal{S} \geqq \mathscr{D}+O(R)$ and $\mathscr{D} \geqq \mathscr{F}$, from which Lemmas follow immediately. Now,

$$
\mathcal{S} \geqq \int_{x}^{2 x-\Delta}=\sum_{x<m, n \leqq 2 x} a_{m} \bar{a}_{n} \text { meas. }\left\{\begin{array}{ll}
t \leqq t \leqq 2 x-\Delta \\
t: \begin{array}{l} 
\\
m-\Delta \leqq t<m \\
n-\Delta \leqq t<n
\end{array}
\end{array}\right\} .
$$

We note that meas. $\{t\}=0$ for $|m-n| \geqq \Delta$. If $x+\Delta<m, n \leqq 2 x-\Delta$ then meas. $\{t\}=\Delta-|m-n|$. Otherwise, the number of $(m, n)$ is $O\left(\Delta^{2}\right)$. Also, meas. $\{t\} \leqq \Delta$ trivially. We thus have

$$
\begin{aligned}
\mathcal{S} & \geqq \sum_{\substack{x+\Delta<m, n \leqq 2 x-\Delta \\
|m-n| \leqq \Delta}} a_{m} \bar{a}_{n}(\Delta-|m-n|)+O(R) \\
& =\sum_{x<m, n \leqq 2 x} \sum_{m} \bar{a}_{n} \max (0, \Delta-|m-n|)+O(R) \\
& =\mathscr{D}+O(R) .
\end{aligned}
$$

Moreover,

$$
\begin{aligned}
\mathscr{D} & =\int_{-\infty}^{\infty} K(\beta, \Delta)\left|\sum_{x<n \leqq 2 x} a_{n} e(\beta n)\right|^{2} d \beta \\
& \geqq \int_{-1 / 2 \Delta}^{1 / 2 \Delta}\left(\frac{\Delta}{2}\right)^{2}\left|\sum_{x<n \leqq 2 x} a_{n} e(\beta n)\right|^{2} d \beta \\
& =\mathscr{F},
\end{aligned}
$$

since $K(y, \Delta) \geqq(\Delta / 2)^{2}$ for $|y| \leqq 1 / 2 \Delta$.

$\mathscr{I}$ reminds us of Circle method or Saddle point method. Also $\mathscr{D}$, Dispersion method or Kloostermania. $\mathcal{S}$, Large sieve or Dirichlet polynomial methods. This observation makes a feature of our argument below. 


\section{Proof of Theorem.}

Let $x$ be a sufficiently large parameter. We take $y=Y^{\theta_{1}}\left(1 / 4<\theta_{1}<1 / 2\right)$ and $Y=x^{\theta_{2}}\left(7 / 12<\theta_{2}<2 / 3\right)$, so that $y=x^{\theta}, 7 / 48<\theta<1 / 3$. Put $\mathcal{K}=(x, x+y], \mathcal{A}=$ $(2 Y, 4 Y]$ and $\mathscr{B}=(x-3 Y, x-2 Y]$. Our aim is to give a lower bound of the sum

$$
\mathcal{G}=\mathcal{G}(2 k)=\sum_{\substack{2 k=p+p^{\prime} \\ p \in \mathscr{B}}} \log p
$$

for $2 k \in \mathcal{K}$. Moreover, define

$$
\mathcal{S}=\mathcal{S}(2 k, z)=\sum_{\substack{\sum_{2=p+n}=p \in \mathcal{D} \\ p \in \mathcal{A} \\(n, \mathcal{P}(2 k, z))=1}} \log p
$$

where

Suppose that

$$
\mathscr{Q}(2 k, z)=\prod_{\substack{p<2 \\ p \nmid 2 k}} p
$$

$$
(4 Y)^{1 / 3}<z<Y^{1 / 2}
$$

Since $2 k \in \mathcal{K}$ and $p \in \mathscr{B}$ imply $n=2 k-p \in \mathcal{A}, n$ counted by $\mathcal{S}$ has at most two prime factors $\geqq z$. We thus have

where

$$
\mathcal{S}-\mathcal{G}=\sum_{\substack{2 k=p+n \\ p \in \mathcal{B}}}(\log p) \rho^{\prime}(n)
$$

For any $l \in \mathcal{A}$,

$$
\rho^{\prime}(n)=\sum_{\substack{n=p p^{\prime} \\ z \leq p \leq p^{\prime}}} 1
$$

$$
\begin{aligned}
\rho^{\prime}(l) & \leqq \sum_{\substack{l=m n \\
z \leqq m \leq n}} \frac{\Lambda(m) \Lambda(n)}{\log m \log n} \\
& \leqq \sum_{\substack{l=m n \\
\sqrt{2} Y<n \leqq 4 Y / 2}} \frac{\Lambda(m) \Lambda(n)}{\log m \log n} \\
& \leqq \frac{1}{\log (Y / z) \log z} \sum_{\substack{l=m n \\
n \in g}} \Lambda(m) \Lambda(n) \\
& =\Xi \rho(l), \text { say. }
\end{aligned}
$$

Here, $g=(\sqrt{2} Y, 4 Y / z]$. We therefore have our fundamental inequality

$$
\begin{aligned}
\mathcal{S}-\mathcal{G} & \leqq \Xi \sum_{\substack{2 k=p+n \\
p \in \mathcal{A}}}(\log p) \rho(n) \\
& =\Omega, \text { say. }
\end{aligned}
$$

We proceed to evaluate $\Omega$ by means of Circle method. Define

$$
Q=y Q_{1}^{-2}
$$




$$
\begin{gathered}
Q_{1}=(\log Y)^{D} \\
\boldsymbol{M}=\bigcup_{q \leqq Q_{1}} \bigcup_{a=1}^{q} * I_{q, a} \quad I_{q, a}=\left[\frac{a}{q}-\frac{1}{q Q}, \frac{a}{q}+\frac{1}{q Q}\right] \\
\boldsymbol{m}=[1 / Q, 1+1 / Q] \backslash \boldsymbol{M},
\end{gathered}
$$

where $D$ is a constant specified later, and $*$ stands for the restriction $(a, q)=1$. Furtheremore,

$$
\begin{aligned}
& T(\alpha)=\Xi \sum_{n \in \mathcal{A}} \rho(n) e(\alpha n), \\
& W(\alpha)=\sum_{p \in \mathcal{B}}(\log p) e(\alpha p) .
\end{aligned}
$$

For $\alpha=a / q+\beta \in I_{q, a}$, we write

$$
V(\alpha)=\Xi \sum_{n \in \mathcal{A}}\left(\rho(n) e\left(\frac{a}{q} n\right)-\Gamma \frac{\mu(q)}{\varphi(q)}\right) e(\beta n)
$$

where

$$
\Gamma=\frac{1}{2} \log \left(\frac{8 Y}{z^{2}}\right)
$$

Then,

$$
\begin{aligned}
\Omega= & \int_{1 / Q}^{1+1 / Q} T(\alpha) W(\alpha) e(-2 k \alpha) d \alpha \\
= & \int_{M}(T(\alpha)-V(\alpha)) W(\alpha) e(-2 k \alpha) d \alpha \\
& +\int_{M} V(\alpha) W(\alpha) e(-2 k \alpha) d \alpha \\
& +\int_{m} T(\alpha) W(\alpha) e(-2 k \alpha) d \alpha \\
= & \Omega_{0}-\Re_{1}-\Re_{2}, \text { say. }
\end{aligned}
$$

We first consider $\Omega_{0}$.

$$
\begin{aligned}
\Omega_{0}= & \sum_{q \leq Q_{1}} \sum_{a=1}^{q} * \int_{-1 / q Q}^{1 / q Q} \Xi \Gamma \frac{\mu(q)}{\varphi(q)}\left(\sum_{n \in \mathcal{A}} e(\beta n)\right)\left(\sum_{p \in \mathcal{B}}(\log p) e\left(\left(\frac{a}{q}+\beta\right) p\right)\right) \\
& \times e\left(-2 k\left(\frac{a}{q}+\beta\right)\right) d \beta \\
= & \Xi \Gamma \sum_{q \leq Q_{1}} \frac{\mu(q)}{\varphi(q)} \sum_{p \in \mathcal{B}}(\log p) c_{q}(p-2 k) \cdot \sum_{n \in \mathcal{A}} \int_{-1 / 2}^{1 / 2} e((n+p-2 k) \beta) d \beta \\
& +O\left(\Xi \Gamma \sum_{q \leq Q_{1}} \frac{|\mu(q)|}{\varphi(q)} \sum_{a=1}^{q} * \int_{1 / q Q}^{1 / 2}\left|\sum_{n \in \mathcal{A}} e(\beta n)\right|\left|W\left(\frac{a}{q}+\beta\right)\right| d \beta\right) .
\end{aligned}
$$

The above $O$-term is 


$$
\ll \mathcal{L}^{-1} \sum_{q \leq Q_{1}}(q Q \cdot Y \mathcal{L})^{1 / 2}
$$

$2 k \in \mathcal{K}$ and $p \in \mathscr{B}$ imply $2 k-p \in \mathcal{A}$, namely

$$
\sum_{n \in \mathcal{A}} \int_{-1 / 2}^{1 / 2} e((n+p-2 k) \beta) d \beta \equiv 1 \text {. }
$$

Hence the main contribution of $\Omega_{0}$ is equal to

$$
\begin{aligned}
& \Xi \Gamma \sum_{q \leq Q_{1}} \frac{\mu(q)}{\varphi(q)} \sum_{\substack{d \mid q \\
(d, 2 k)=1}} d \mu\left(\frac{q}{d}\right) \sum_{\substack{p \in \mathcal{p} \\
p=2 k(d)}} \log p+O\left(\mathcal{L}^{-1} \sum_{q \leq Q_{1}} \frac{|\mu(q)|}{\varphi(q)} \sum_{d \mid q} d \mathcal{L}^{2}\right) \\
& =\Xi \Gamma \sum_{q \leq Q_{1}} \frac{\mu(q)}{\varphi(q)} \sum_{\substack{d, q \\
(d, 2 k)=1}} d \mu\left(\frac{q}{d}\right) \frac{Y}{\varphi(d)} \\
& +O\left(\mathcal{L}^{-1} \sum_{q \leq Q_{1}} \frac{|\mu(q)|}{\varphi(q)} \sum_{\substack{d \leq q \\
(d, 2 k)=1}} d\left|\sum_{\substack{p \in \mathcal{G} \\
p \equiv 2 k(d)}} \log p-\frac{Y}{\varphi(d)}\right|\right)+O\left(Q_{1} \mathcal{L}^{2}\right) \\
& =Y \Xi \Gamma \sum_{q \leqq Q_{1}} \frac{\mu^{2}(q)}{\varphi^{2}(q)} c_{q}(2 k) \\
& +O\left(\sum_{\substack{d \leq Q \\
(d, 2 k)=1}}\left|\sum_{\substack{x-3 Y<p \leq x-2 Y \\
p \equiv 2 k(d)}} \log p-\frac{Y}{\varphi(d)}\right|+Y^{3 / 4}\right) .
\end{aligned}
$$

By Lemmas 2 and 3 , the above $O$-term is

$$
\ll Y \mathcal{L}^{-E},
$$

for any $E>0$. Put

$$
\mathfrak{R}_{0}=\mathfrak{R}_{0}(2 k)=Y \Xi \Gamma \sum_{q>Q_{1}} \frac{\mu^{2}(q)}{\varphi^{2}(q)} c_{q}(2 k) .
$$

Then, by [15, Kap. VI p. 201], we see

$$
\sum_{2 \in \in, \mathcal{K}} \Re_{0}(2 k)^{2} \ll y Y^{2} Q_{1}^{-1} .
$$

In conjuction with (3.4)-(3.7) we obtain

$$
\Omega_{0}=\Im(2 k) Y \Xi \Gamma-\mathfrak{R}_{0}+O\left(Y \mathcal{L}^{-3}\right)
$$

where

$$
\Im(2 k)=2 \prod_{p>2}\left(1-\frac{1}{(p-1)^{2}}\right) \prod_{\substack{p \backslash k \\ p>2}}\left(\frac{p-1}{p-2}\right) .
$$

Next we consider $\mathfrak{R}_{1}$. Let $I_{q^{\prime}, a^{\prime}}, I_{\boldsymbol{q}, a} \subset \boldsymbol{M}, a^{\prime} / q^{\prime} \neq a / q$. If $\alpha^{\prime} \in I_{q^{\prime}, a^{\prime}}$ and $\alpha \in$ $I_{q, a}$ then we find 


$$
\begin{aligned}
\left\|\alpha^{\prime}-\alpha\right\| & \geqq\left\|\frac{a^{\prime}}{q^{\prime}}-\frac{a}{q}\right\|-\left\|\alpha^{\prime}-\frac{a^{\prime}}{q^{\prime}}\right\|-\left\|\alpha-\frac{a}{q}\right\| \\
& \geqq \frac{1}{q^{\prime} q}-\frac{1}{q^{\prime} Q}-\frac{1}{q Q} \\
& \geqq\left(2 Q_{1}\right)^{-2} .
\end{aligned}
$$

We therefore have

$$
\begin{aligned}
& \sum_{2 \in \in \mathcal{K}^{\prime}} \Re_{1}(2 k)^{2} \\
& \ll \int_{M} \int_{M}\left|V\left(\alpha^{\prime}\right) W\left(\alpha^{\prime}\right)\right||V(\alpha) W(\alpha)| \min \left(y, \frac{1}{\left\|\alpha^{\prime}-\alpha\right\|}\right) d \alpha^{\prime} d \alpha \\
& \ll Q_{1}{ }^{2}\left(\int_{M}|V(\alpha) W(\alpha)| d \alpha\right)^{2}+y \sum_{q \leq Q_{1}} \sum_{a=1}^{q} *\left(\int_{I_{q, a}}|V(\alpha) W(\alpha)| d \alpha\right)^{2} \\
& \ll{Q_{1}}^{2} \int_{M}|V(\alpha)|^{2} d \alpha \int_{M}|W(\alpha)|^{2} d \alpha \\
& \quad+y \sup _{\substack{\alpha, q)=1 \\
q \leq Q_{1}}} \int_{I_{q, a}}|V(\alpha) W(\alpha)| d \alpha \cdot \int_{M}|V(\alpha) W(\alpha)| d \alpha \\
& \ll Q_{1}{ }^{2} Y^{2} \mathcal{L}+y Y^{3 / 2} \mathcal{L} \sup _{\substack{\alpha, q)=1 \\
q \leq Q_{1}}}\left(\int_{I_{q, a}}|V(\alpha)|^{2} d \alpha\right)^{1 / 2},
\end{aligned}
$$

by Cauchy's inequality. Here we appeal to the following lemma. We shall prove Lemma 9 in the next section.

LemmA 9. Let $\varepsilon, B, E>0$ be given. If $Y^{1 / 4+\varepsilon} \leqq \Delta \leqq Y^{1 / 2}$ and $4 Y / z \leqq Y^{8 / 15}$ then

$$
\Delta^{2} \int_{-1 / 2 \Delta}^{1 / 2 \Delta}\left|V\left(\frac{a}{q}+\beta\right)\right|^{2} d \beta \ll \Delta^{2} Y \mathcal{L}^{-E}
$$

uniformly for $(a, q)=1$ and $q \leqq(\log Y)^{B}$. Here the implied constant depends only on $\varepsilon, B$ and $E$.

Now, we take $2 \Delta=Q$ and

$$
z=Y^{7 / 15+\delta}
$$

with a sufficiently small $\delta>0$. Then this choice of $z$ satisfies the assumption of Lemma 9, also (3.1). We thus obtain

$$
\sum_{k \in \mathcal{K}} \Re_{1}(2 k)^{2} \ll y Y^{2} \mathcal{L}^{-E}
$$

for any $E>0$.

We turn to $\Re_{2}$. For $2 \Delta<y$, we have 


$$
\begin{aligned}
& \sum_{2 \in \in} \Re_{2}(2 k)^{2} \ll \int_{m} \int_{m}\left|T\left(\alpha^{\prime}\right) W\left(\alpha^{\prime}\right)\right||T(\alpha) W(\alpha)| \min \left(y, \frac{1}{\left\|\alpha^{\prime}-\alpha\right\|}\right) d \alpha^{\prime} d \alpha \\
& \ll \Delta\left(\int_{m}|T(\alpha) W(\alpha)| d \alpha\right)^{2} \\
& +y \int_{m}|T(\alpha) W(\alpha)|\left(\int_{m_{\left\|\alpha^{\prime}-\alpha\right\| \leq 1 / 2 \Delta}}\left|T\left(\alpha^{\prime}\right) W\left(\alpha^{\prime}\right)\right| d \alpha^{\prime}\right) d \alpha \\
& \ll \Delta \int_{m}|T(\alpha)|^{2} d \alpha \int_{m}|W(\alpha)|^{2} d \alpha \\
& +y\left(\sup _{\alpha \in m} \int_{\left\|\alpha^{\prime}-\alpha\right\| \leq 1 / 2 \Delta}\left|T\left(\alpha^{\prime}\right)\right|^{2} d \alpha^{\prime}\right)^{1 / 2}\left(\int_{m}|T(\alpha)|^{2} d \alpha\right)^{1 / 2} \int_{m}|W(\alpha)|^{2} d \alpha \\
& \ll \Delta Y^{2} \mathcal{L}^{4}+y Y^{3 / 2} \mathcal{L}^{3} \sup _{\alpha \in m}\left(\int_{-1 / 2 \Delta}^{1 / 2 \Delta}|T(\alpha+\beta)|^{2} d \beta\right)^{1 / 2},
\end{aligned}
$$

since $T(\alpha)$ has the period 1 .

We now use the following lemma. We postpone the proof of Lemma 10 until the final section.

LEMmA 10. Let $1<\Delta<Y^{1 / 2}$. Suppose that $|\alpha-a / q| \leqq 1 / q^{2}$ with $(a, q)=1$. Let $E, \varepsilon>0$ be given. If $4 Y / z \leqq Y^{8 / 15-\varepsilon}$ then

$$
\Delta^{2} \int_{-1 / 2 \Delta}^{1 / 2 \Delta}|T(\alpha+\beta)|^{2} d \beta \ll \Delta Y \mathcal{L}^{F}\left\{Y^{1 / 4}+\Delta q^{-1 / 2}+(q \Delta)^{1 / 2}\right\}+\Delta^{2} Y \mathcal{L}^{-E}
$$

where the implied constant depends only on $E$ and $\varepsilon$.

We take $\Delta=y Q_{1}^{-1}=Q Q_{1}$. The assumption of Lemma 10 is satisfied, because of (3.10). For any $\alpha \in \boldsymbol{m}$ there exist $a$ and $q$ such that $|\alpha-a / q| \leqq 1 / q^{2},(a, q)=1$ and $Q_{1}<q \leqq Q$. Thus, Lemma 10 yields

$$
\begin{aligned}
\sup _{\alpha \in m} \Delta^{2} \int_{-1 / 2 \Delta}^{1 / 2 \Delta}|T(\alpha+\beta)|^{2} d \beta & \ll \sup _{Q_{1}<q \leq Q} \Delta Y \mathcal{L}^{F}\left\{Y^{1 / 4}+\Delta q^{-1 / 2}+(q \Delta)^{1 / 2}\right\}+\Delta^{2} Y \mathcal{L}^{-E} \\
& \ll \Delta^{2} Y \mathcal{L}^{F} Q_{1}^{-1 / 2} .
\end{aligned}
$$

Combining this with (3.12) we obtain

$$
\sum_{2 \in \in \mathcal{K}} \Re_{2}(2 k)^{2} \ll y Y^{2} \mathcal{L}^{F} Q_{1}^{-1 / 4} .
$$

For any given $E>0$, we take $D=4(E+F)$. Then $(3.8)$, (3.11) and (3.13) become

$$
\sum_{i=0}^{2} \sum_{2 \in \in \mathcal{K}} \Re_{i}(2 k)^{2} \ll y Y^{2} \mathcal{L}^{-E}
$$

Finally we calculate $\Xi \Gamma$. Because of $(3.10)$, 


$$
\begin{aligned}
(\log Y) \Xi \Gamma & =\frac{(1 / 2) \log \left(8 Y / z^{2}\right) \cdot(\log Y)}{\log (Y / z) \cdot \log z} \\
& \leqq \frac{(1 / 2) \cdot(1 / 15)}{(8 / 15) \cdot(7 / 15)}+\delta_{1} \\
& <\frac{1}{7}+\delta_{1}
\end{aligned}
$$

for sufficiently large $Y$ and small $\delta_{1}$. In conjunction with (3.3), (3.9), (3.14) and (3.15) we therefore have

PROPOSITION 1.

$$
\Omega \leqq\left(\frac{1}{7}+\delta_{1}\right) \subseteq(2 k) \frac{Y}{\log Y}-\sum_{i=0}^{2} \Re_{i}+O\left(Y(\log Y)^{-3}\right) .
$$

Here, $\Re_{i}^{\prime}$ s satisfy (3.14).

In the next stage of the proof we use Sieve method. We remember

$$
\mathcal{S}=\sum_{\substack{2 k=p+n \\ 2 k \in \mathscr{Q} \\(n, \mathscr{P}(2 k, z))=1}} \log p=\sum_{\substack{p \in \mathscr{S} \\(2 k-p, \mathcal{P}(2 k, z))=1}} \log p .
$$

For $d$ with $(d, 2 k)=1$, put

$$
\begin{aligned}
r(\mathscr{B}, d, 2 k) & =\sum_{\substack{p \in \mathscr{B} \\
p=2 k(d)}} \log p-\frac{Y}{\varphi(d)} \\
& =\sum_{\substack{2 k=p+n \\
p=\mathbb{P} \\
d \mid n}} \log p-\frac{Y}{\varphi(d)} .
\end{aligned}
$$

Then Lemma 5 yields, on taking $D=Y^{1-\delta}>z^{2}$,

$$
\begin{aligned}
\mathcal{S} \geqq & Y \prod_{\substack{p<2 \\
p \nmid 2 k}}\left(1-\frac{1}{\varphi(p)}\right)\left\{f\left(\frac{\log D}{\log z}\right)+O\left((\log \mathcal{L})^{-1}\right)\right\} \\
& +\sum_{\substack{(d, 2 k)=1 \\
d<D}} \lambda_{d} r(\mathscr{B}, d, 2 k) \\
& =\Pi+\Sigma, \text { say. }
\end{aligned}
$$

We first consider $\Sigma$.

$$
\begin{aligned}
\sum= & \sum_{\substack{(d, 2 k)=1 \\
d<D}} \lambda_{d} \sum_{\substack{p \in \mathscr{B} \\
p \equiv 2 k(d)}} \log p-Y \sum_{\substack{(d, 2 k)=1 \\
d<D}} \frac{\lambda_{d}}{\varphi(d)} \\
= & \sum_{d<D} \lambda_{d} \sum_{\substack{2 k=p+n \\
p \in \mathcal{B} \\
d \mid n}} \log p-Y \sum_{d<D} \frac{\lambda_{d}}{d} \sum_{q \backslash d} \frac{\mu(q)}{\varphi(q)} c_{q}(2 k) \\
& +O\left(\sum_{\substack{(, 2 k)>1 \\
d<D}}\left|\lambda_{d}\right| \sum_{\substack{p \in \mathscr{p} \\
p \equiv 2 k(d)}} \log p\right)
\end{aligned}
$$




$$
\begin{aligned}
= & \sum_{\substack{2 k=p+n \\
p \in \mathscr{B}}}(\log p)\left(\sum_{\substack{d \uparrow n \\
d<D}} \lambda_{d}\right)-Y \sum_{q \leq Q_{1}} \frac{\mu(q)}{\varphi(q)}\left(\sum_{\substack{d<n \\
q \backslash d}} \frac{\lambda_{d}}{d}\right) c_{q}(2 k) \\
& +O\left(D \mathcal{L}^{2}+Y \mathcal{L} \sum_{Q_{1}<q<D} \frac{|\mu(q)|}{\varphi(q) q}\left|c_{q}(2 k)\right|\right) \\
= & Z-Z_{1}+\Re_{3}, \text { say. }
\end{aligned}
$$

By $[15$, Kap. VI], as before, we see

$$
\sum_{2 \in \mathscr{B}} \Re_{3}(2 k)^{2} \ll y Y^{2} \mathcal{L}^{4} Q_{1}^{-1}
$$

We next appeal to Circle method. Put

For $\alpha=a / q+\beta \in I_{q, a}$, we write

$$
S(\alpha)=\sum_{n \in \mathcal{A}}\left(\sum_{\substack{d<n \\ d<D}} \lambda_{d}\right) e(\alpha n)
$$

$$
U(\alpha)=\sum_{n \in \mathcal{A}}\left(\left(\sum_{\substack{d \backslash n \\ d<D}} \lambda_{d}\right) e\left(\frac{a}{q} n\right)-\left(\sum_{\substack{d<D \\ q \backslash d}} \frac{\lambda_{d}}{d}\right)\right) e(\beta n) .
$$

We then have

$$
\begin{aligned}
Z= & \int_{1 / Q}^{1+1 / Q} S(\alpha) W(\alpha) e(-2 k \alpha) d \alpha \\
= & \int_{M}(S(\alpha)-U(\alpha)) W(\alpha) e(-2 k \alpha) d \alpha \\
& +\int_{M} U(\alpha) W(\alpha) e(-2 k \alpha) d a \\
& +\int_{m} S(\alpha) W(\alpha) e(-2 k \alpha) d \alpha \\
= & Z_{0}+\Re_{4}+\Re_{5}, \quad \text { say. }
\end{aligned}
$$

By the argument similar to that for $\Omega$, we infer that

$$
\begin{gathered}
Z_{0}=Z_{1}+O\left(Y \mathcal{L}^{-3}\right), \\
\sum_{2 \in \mathcal{K}^{\prime}} \Re_{4}(2 k)^{2} \ll{Q_{1}}^{2} Y^{2} \mathcal{L}^{4}+y Y^{3 / 2} \mathcal{L}^{3} \sup _{\substack{(\alpha, q)=1 \\
q \leq Q_{1}}}\left(\int_{I_{q, a}}|U(\alpha)|^{2} d \alpha\right)^{1 / 2}, \\
\sum_{2 \in \mathcal{K}^{\prime}} \Re_{5}(2 k)^{2} \ll \Delta Y^{2} \mathcal{L}^{4}+y Y^{3 / 2} \mathcal{L}^{3} \sup _{\alpha \in m}\left(\int_{-1 / 2 \Delta}^{1 / 2 \Delta}|S(\alpha+\beta)|^{2} d \beta\right)^{1 / 2} .
\end{gathered}
$$

We now use the following lemmas, which will be proven later.

LEMMA 11. In Lemma 9, replace the sum $V$ and the condition $4 Y / z \leqq Y^{8 / 15}$ by $U$ and $D \leqq Y^{1-\varepsilon}$, respectively. Then, the resulting assertion holds true.

LEMMA 12. In Lemma 10, replace the sum $T$ and the condition $4 Y / z \leqq Y^{8 / 15-\varepsilon}$ 
by $S$ and $D \leqq Y^{1-\varepsilon}$, respectively. Then, the resulting assertion holds true.

On taking the parameters as before, we find

$$
\sum_{i=3}^{5} \sum_{k \in \mathcal{K}} \Re_{i}(2 k)^{2} \ll y Y^{2} \mathcal{L}^{-E}
$$

for any $E>0$. In conjunction with (3.17)-(3.20) we obtain

$$
\Sigma=\sum_{i=3}^{5} \Re_{i}+O\left(Y \mathcal{L}^{-3}\right)
$$

We turn now to $\Pi$. By Mertens' theorem,

$$
\begin{aligned}
\prod_{\substack{p<z \\
p \nmid 2 k}}\left(1-\frac{1}{\varphi(p)}\right) & =\prod_{2<p<z}\left(\frac{p-1}{p} \frac{p(p-2)}{(p-1)^{2}}\right) \cdot \prod_{\substack{2<p_{<2} \\
p \backslash k}}\left(\frac{p-1}{p-2}\right) \\
& =\prod_{p<z}\left(1-\frac{1}{p}\right) \cdot 2 \prod_{2<p<z}\left(1-\frac{1}{(p-1)^{2}}\right) \prod_{\substack{p \mid k \\
2<p<z}}\left(\frac{p-1}{p-2}\right) \\
& =\prod_{p<z}\left(1-\frac{1}{p}\right) \cdot 2 \prod_{2<p<z}\left(1-\frac{1}{(p-1)^{2}}\right) \prod_{\substack{p<k \\
2<p<z}}\left(\frac{p-1}{p-2}\right) \\
& =\frac{e^{-\gamma}}{\log z} \Im(2 k)\left(1+O\left((\log z)^{-1}\right)\right) .
\end{aligned}
$$

Thus,

$$
\begin{aligned}
\Pi & =\Im(2 k) Y \frac{e^{-\gamma}}{\log z} \frac{2 e^{\gamma} \log (\log D / \log z-1)}{\log D / \log z}\left(1+O\left((\log \log Y)^{-1}\right)\right) \\
& =\subseteq(2 k) Y \cdot K, \text { say. }
\end{aligned}
$$

Since $\log x>1-1 / x$ for $x>1$, we see

$$
\begin{aligned}
(\log Y) K & =\frac{2 \log (\log D / \log z-1)}{\log D / \log Y}\left(1+O\left((\log \log Y)^{-1}\right)\right) \\
& \geqq 2 \log \left(\frac{15}{7}-1\right)-\delta_{2} \\
& >\frac{1}{4}-\delta_{2}
\end{aligned}
$$

for sufficiently large $Y$ and small $\delta_{2}$. Hence, (3.23) becomes

$$
\Pi>\left(\frac{1}{4}-\boldsymbol{\delta}_{2}\right) \frac{\mathfrak{S}(2 k) Y}{\log Y} \text {. }
$$

Combining this with (3.16) and (3.22) we therefore obtain

PROPOSITION 2. 


$$
\mathcal{S}>\left(\frac{1}{4}-\delta_{2}\right) \subseteq(2 k) \frac{Y}{\log Y}+\sum_{i=3}^{5} \Re_{i}+O\left(Y(\log Y)^{-3}\right) .
$$

Here $\mathfrak{R}_{i}$ 's satisfy (3.21).

In conjunction with (3.2), Propositions 1 and 2 , we finally have

$$
\begin{aligned}
\sum_{\substack{k=p_{+} \\
p \in \mathcal{B}}} \log p & >\mathcal{S}(2 k)-\Omega(2 k) \\
& >\left(\frac{1}{4}-\frac{1}{7}-\left(\delta_{1}+\delta_{2}\right)\right) \subseteq(2 k) \frac{Y}{\log Y}+\sum_{i=0}^{5} \Re_{i}(2 k)+O\left(Y(\log Y)^{-3}\right) \\
& >\frac{S(2 k) Y}{10 \log Y}+R(2 k)
\end{aligned}
$$

for sufficiently large $Y$. Here, $R(2 k)=\sum_{i=0}^{5} \Re_{i}(2 k)$.

We shall derive Theorem from (3.22), (3.14) and (3.19). We first note $\Im(2 k)>c_{5}$, see $[15$, Kap. VI]. Let $\mathcal{E}$ denote the exceptional set in Goldbach's problem. For all $2 k \in \mathcal{E}$, the left hand side of (3.22) is zero. If $|R(2 k)| \leqq$ $\Im(2 k) Y / 11 \log Y$ for some $2 k \in \mathcal{K}=(x, x+y]$, then the right hand side of $(3.22)$ is posititive, whence $2 k \notin \mathcal{E} \cap \mathcal{K}$. Consecuently, it follows from (3.14) and (3.19) that, for any $A>0$,

or

$$
\begin{aligned}
\# \mathcal{E} \cap \mathcal{K}\left(\frac{Y}{\log Y}\right)^{2} & \ll \sum_{2} \sum_{k \in \mathcal{E} \cap \mathcal{K}}|R(2 k)|^{2} \\
& \leqq \sum_{2 \in \mathcal{K}}|R(2 k)|^{2} \\
& \ll y Y^{2} \mathcal{L}^{-(A+2)}
\end{aligned}
$$

$$
E(x+y)-E(x)=\# \mathcal{E} \cap \mathcal{K} \ll y \mathcal{L}^{-A},
$$

as required.

This completes our proof of Theorem, apart from the verification of Lemmas $9,10,11$ and 12 .

\section{Major arc.}

Let $\varepsilon^{\prime}, B>0$ be given. Throughout this section we assume

$$
x^{1 / 4+\varepsilon^{\prime}} \leqq \Delta \leqq x^{1 / 2}, \quad(a, q)=1, \quad q \leqq(\log x)^{B} .
$$

We use the convention

$$
|f|_{i}=\int_{x}^{2 x}|f(t, \Delta, q, a)|^{i} d t, \quad i=1,2 .
$$

We call $f(t, \Delta, q, a)$ "admissible" if for any $E>0$ 


$$
|f|_{2} \ll \Delta^{2} x \mathcal{L}^{-E}
$$

where the implied constant depends only on $\varepsilon^{\prime}, B$ and $E$. An admissible function is abbreviated to "A.R." in a formula. Under the assumption (4.1) we shall show Lemmas $9^{\dagger}$ and $11^{\dagger}$ below. By Lemma 7, Lemmas 9 and 11 follow from Lemmas $9^{\dagger}$ and $11^{\dagger}$, respectively.

LEMMA $9^{\dagger}$. Define

$$
v=v(t, \Delta, q, a ; M, N)=\sum_{\substack{t<m n \leq t+\Delta \\ M<n \leq N}} \Lambda(m) \Lambda(n) e\left(\frac{a}{q} m n\right) .
$$

If $x^{1 / 2} \leqq M<N \leqq x^{8 / 15}$ then

$$
v=\frac{\mu(q)}{\varphi(q)} \Delta \log \left(\frac{N}{M}\right)+A . R .
$$

ProOF. The terms with $(m n, q)>1$ contribute to $v$ at most

$$
\begin{aligned}
\sum_{\substack{(m, q)>1 \\
t<m n \sum t+1 \\
x^{2} \ll m, n \ll x^{1-\varepsilon}}} \Lambda(m) \Lambda(n) & \ll \sum_{\substack{x^{\varepsilon} \ll m \ll x^{1-\varepsilon} \\
(m, q)>1}} \Lambda(m)\left(\frac{\Delta}{m}+1\right) \mathcal{L} \\
& \ll\left(\Delta x^{-\varepsilon}+1\right) \mathcal{L}^{3},
\end{aligned}
$$

which is admissible trivially. We then have

$$
v=\frac{1}{\varphi(q)} \sum_{\chi(q)} \chi(a) \tau(\bar{\chi}) \sum_{\substack{t<m \leq t+\Delta \\ n \in \mathcal{G}}} \chi(m n) \Lambda(m) \Lambda(n)+A . R . .
$$

Here $g=(M, N]$. By the explicit formula [15, Kap. VII Satz 4.4.],

$$
\begin{aligned}
& \sum_{t / n<m \leq(t+\Delta) / n} \chi(m) \Lambda(m)=E_{0} \frac{\Delta}{n} \\
&-\sum_{\substack{\rho(x) \\
\mid \operatorname{Im}(\rho) \backslash \leq x^{2}}} \frac{(t+\Delta)^{\rho}-t^{\rho}}{n^{\rho} \rho} \\
&+ O\left(\frac{x / n}{x^{2}}(\log q x)^{2}+(\log q x) \sum_{j=0,1} \min \left(1, \frac{x / n}{x^{2}\|(t+j \Delta) / n\|}\right)\right)
\end{aligned}
$$

where $E_{0}=1$ when $\chi$ is the principal character and $E_{0}=0$ otherwise. Thus,

$$
\begin{aligned}
v= & \frac{\mu(q)}{\varphi(q)} \Delta \sum_{\substack{n \in \mathcal{G} \\
(n, q)=1}} \frac{\Lambda(n)}{n} \\
& -\frac{1}{\varphi(q)} \sum_{\chi(q)} \chi(a) \tau(\bar{\chi}) \sum_{\substack{\rho(\mathcal{}) \\
|\operatorname{Im}(\rho)| \leq x^{2}}} \frac{(t+\Delta)^{\rho}-t^{\rho}}{\rho} \sum_{n \in \mathcal{I}} \frac{\chi(n) \Lambda(n)}{n^{\rho}} \\
& +O\left(\sqrt{q}\left(\mathcal{L}^{3} x^{-1}+\mathcal{L}^{2} \sum_{j=0,1} \sum_{n \in \mathcal{I}} \min \left(1, \frac{1 / x}{n\|(t+j \Delta) / n\|}\right)\right)\right)+A \cdot R .
\end{aligned}
$$




$$
\begin{aligned}
= & \frac{\mu(q)}{\varphi(q)} \Delta \log \left(\frac{N}{M}\right) \\
& -\frac{1}{\varphi(q)} \sum_{\chi(q)} \chi(a) \tau(\bar{\chi})\left(\sum_{\substack{\rho(\chi) \\
|\operatorname{m}(\rho)| \leq x}}+\sum_{\substack{\rho(\chi) \\
x<\operatorname{lm}(\rho) \mid \leq x^{2}}}\right) \theta(\rho) N(\rho, \chi) \\
& +O\left(\sqrt{q} \mathcal{L}^{2} \sum_{j=0,1} \sum_{n \in \mathcal{g}} \min \left(1, \frac{1}{n x\|(t+j \Delta) / n\|}\right)\right)+A . R . \\
= & v_{0}-\left(v_{1}+v_{2}\right)+O\left(v_{3}\right)+A . R ., \text { say, }
\end{aligned}
$$

where

$$
\begin{gathered}
N(s, \chi)=\sum_{n \in \mathcal{I}} \chi(n) \Lambda(n) n^{-s}, \\
\theta(s)=\theta(s, t, \Delta)=\frac{(t+\Delta)^{s}-t^{s}}{s} .
\end{gathered}
$$

First we consider $v_{3}$.

$$
\begin{aligned}
\left|v_{3}\right|_{1} & \ll \sqrt{q} \mathcal{L}^{2} \sum_{n \in \mathcal{I}} \int_{x}^{3 x} \min \left(1, \frac{1}{n x\|t / n\|}\right) d t \\
& \ll \sqrt{q} \mathcal{L}^{2} \sum_{n \in \mathcal{I}} x^{-1} \int_{x / n}^{3 x / n} \min \left(n x, \frac{1}{\|u\|}\right) d u \\
& \ll \sqrt{q} \mathcal{L}^{4} .
\end{aligned}
$$

Since $v_{3} \ll \sqrt{q} \mathcal{L}^{2} N$ trivially, we have

$$
\left|v_{3}\right|_{2} \ll q N \mathcal{L}^{6} .
$$

Hence $v_{3}$ is admissible.

We proceed to $v_{2}$. We note that the number of $\rho$ with $T<|\operatorname{Im}(\rho)| \leqq T+1$ is $O(\log T)$. By partial summation and Lemma 4, we then have

$$
\begin{aligned}
& \left|v_{2}\right|_{2} \ll \mathcal{L} \sum_{\chi(q)} \int_{x}^{2 x}\left|\sum_{\substack{\rho\left(\operatorname{Ix}_{\mathrm{m}}\right) \mid \leq x^{2}\\
}} \frac{t^{\rho}}{\rho} N(\rho, \chi)\right|^{2} d t \\
& \ll \mathcal{L} \sum_{\chi(q)} \sum_{\substack{\rho(x) \\
x<\operatorname{Im}(\rho) \mid \leq x^{2}}} \frac{x^{1+2 \operatorname{Re}(\rho)}}{|\rho|^{2}}|N(\rho, \chi)|^{2} \sum_{\substack{\rho^{\prime}(x) \\
\operatorname{Im}\left(\rho^{\prime}\right) \mid \leq x^{2}}} \frac{1}{1+\left|\rho^{\prime}+\bar{\rho}\right|} \\
& \ll \mathcal{L}^{5} \sup _{\substack{x \leq U \leq x^{2} \\
0 \leq \sigma \leq 1}} \frac{x^{1+2 \sigma}}{U^{2}}(q U+N) M^{1-2 \sigma} \mathcal{L}^{12} \\
& \left.\ll \mathcal{L}^{17} \sup _{\sigma}\left(q M\left(\frac{x}{N}\right)^{2 \sigma}+N \frac{x}{M}\right)^{2 \sigma-1}\right) \\
& \ll q x^{3 / 2} \mathcal{L}^{17} \text {. }
\end{aligned}
$$

Because of (4.1), $v_{2}$ is also admissible. 
We turn now to $v_{1}$. We write $\theta(\rho)=\int_{1}^{1+\Delta / t} u^{\rho-1} d u \cdot t^{\rho}$ for $|\operatorname{Im}(\rho)| \leqq x / \Delta$. On splitting the remaining range of $|\operatorname{Im}(\rho)|$ into intervals $\left((x / \Delta) 2^{j},(x / \Delta) 2^{j+1}\right]$, we use Cauchy's inequality. Thus,

$$
\begin{aligned}
\left|\sum_{\rho} \theta(\rho) N(\rho, \chi)\right|_{2} \ll & \left(\frac{\Delta}{x}\right)^{2} \sup _{1 \leqq u \leqq 1+\Delta / x} \int_{x}^{2 x}\left|\sum_{\substack{\rho(x) \\
|\operatorname{Im}(\rho)| \leq x / \Delta}} u^{\rho-1} t^{\rho} N(\rho, \chi)\right|^{2} d t \\
& +\mathcal{L}^{2} \sup _{x / \Delta \leqq U \leqq x / 2} \int_{x}^{3 x}\left|\sum_{\substack{\rho(x) \\
\rho(x) \mid \leq 2 U}} \frac{t^{\rho}}{\rho} N(\rho, \chi)\right|^{2} d t \\
& \ll \mathcal{L}^{4} \sup _{x / \Delta \leq U \leqq x} \sum_{\substack{\rho(\chi) \\
|\operatorname{Im}(\rho)| \leqq U}} \frac{x^{1+2 \operatorname{Re}(\rho)}}{U^{2}}|N(\rho, \chi)|^{2} .
\end{aligned}
$$

Hence, by partial summation,

$$
\begin{aligned}
& \left|v_{1}\right|_{2} \leqq \frac{q}{\varphi(q)} \sum_{\chi(q)}\left|\sum_{\rho(\chi)} \theta N\right|_{2} \\
& \ll \mathcal{L}^{6} \sup _{\substack{x / 4 \leq U \leq x \\
0 \leqq \sigma \leq 1}} \frac{x^{1+2 \sigma}}{U^{2}} \sum_{\chi(q)} \sum_{\substack{\rho(\chi) \\
\operatorname{Re}(\rho) \\
\operatorname{Im}(\rho) \mid \leq U}}|N(\rho, \chi)|^{2} \\
& =\mathcal{L}^{6} \sup _{\substack{x / \Delta \leq U \leq x \\
0 \leq \sigma \leq 1}} x^{1+2 \sigma} U^{-2} K_{\sigma}(U), \text { say . }
\end{aligned}
$$

In case of $0 \leqq \sigma \leqq 1 / 2$ Lemma 4 yields

$$
K_{\sigma}(U) \ll \mathcal{L}^{F}(q U+N) N^{1-2 \sigma},
$$

since $1-2 \sigma, 2-2 \sigma \geqq 0$. Moreover the supremum over $U$ is attained at $U=x / \Delta$. This contributes to $\left|v_{1}\right|_{2}$

$$
\begin{aligned}
& \ll \mathcal{L}^{F} \sup _{0 \leqq \sigma \leqq 1 / 2} \Delta^{2} x^{2 \sigma-1}\left(q \frac{x}{\Delta}+N\right) N^{1-2 \sigma} \\
& \ll \mathcal{L}^{F} \Delta^{2} x \sup _{0 \leqq \sigma \leqq 1 / 2}\left(\frac{q}{\Delta}\left(\frac{x}{N}\right)^{2 \sigma-1}+\left(\frac{x}{N}\right)^{2 \sigma-2}\right) \\
& \ll \Delta^{2} x \mathcal{L}^{F}\left(\frac{q}{\Delta}+\frac{N}{x}\right) \\
& \ll \Delta^{2} x^{3 / 4} .
\end{aligned}
$$

In another case $1 / 2 \leqq \sigma \leqq 1$, Lemma 2 yields that the supremum may be taken over $1 / 2 \leqq \sigma \leqq 1-\eta(x)$ only, where $\eta(x)=c_{4}(\log x)^{-4 / 5}$. Furtheremore, by Lemmas 3 and 4 , we have

$$
\begin{aligned}
K_{\sigma}(U) & \leqq\left(\sum_{\chi(q)} \sum_{\substack{\rho(x) \\
\operatorname{Re}(\rho) \geq \sigma \\
\mid \operatorname{Im}(\rho) ! \leq U}} 1\right)^{1 / 2}\left(\sum_{\chi(q)} \sum_{\substack{\rho(x) \\
\operatorname{Re}(\rho) \geq \sigma \\
|\operatorname{Im}(\rho)| \leq U}}|N(\rho, \chi)|^{4}\right)^{1 / 2} \\
& \ll \mathcal{L}^{F}\left((q U)^{k(1-\sigma)}\right)^{1 / 2}\left(\sup _{M \leqq V \leqq N}\left(q U+V^{2}\right) V^{2(1-2 \sigma)}\right)^{1 / 2}
\end{aligned}
$$


Here $k>12 / 5$. We see the supremum over $U$ is attained at $U=x / \Delta$ again. Then, since $x / \Delta \leqq M^{2}$ and $1-\sigma \geqq 0$, we have that the contribution to $\left|v_{1}\right|_{2}$ is at most

$$
\Delta^{2} x q \mathcal{L}^{F} \sup _{1 / 2 \leq \sigma \leq 1-\eta(x)}\left(\left(\frac{x}{\Delta}\right)^{k / 4} \frac{N}{x}\right)^{2(1-\sigma)}
$$

We now note that

$$
\left(1-\frac{1}{4}\right) \frac{12}{5} \frac{1}{4}+\left(\frac{8}{15}-1\right)=-\frac{1}{60}
$$

Hence, there exists a constant $\xi>0$ such that

$$
\left(\frac{x}{\Delta}\right)^{k / 4} \frac{N}{x} \leqq x^{-\xi}
$$

Thus (4.7) becomes

$$
\begin{aligned}
& \ll \Delta^{2} x q \mathcal{L}^{F} \sup _{1 / 2 \leq \sigma \leq 1-\eta(x)} x^{-2 \xi(1-\sigma)} \\
& \ll \Delta^{2} x q \mathcal{L}^{F} \exp \left(-c_{6}(\log x)^{-1 / 5}\right) \\
& \ll \Delta^{2} x \mathcal{L}^{-E}
\end{aligned}
$$

for any $E>0$.

In conjunction with (4.5), (4.6) and (4.8), $v_{1}$ is admissible. Combining this with (4.2), (4.3) and (4.4), we conclude that $v-v_{0}$ is also admissible, as required.

LEMMA $11^{\dagger}$. Define, for arbitrary sequence $\left(\lambda_{d}\right)$ with $\left|\lambda_{d}\right| \leqq 1$,

$$
u=u(t, \Delta, q, a ; D)=\sum_{t<n \leq t+\Delta}\left(\sum_{\substack{d \backslash n \\ d<D}} \lambda_{d}\right) e\left(\frac{a}{q} n\right) .
$$

For any $\varepsilon>0$, if $D \leqq x^{1-\varepsilon}$ then

$$
u=\left(\sum_{\substack{d<D \\ q \backslash d}} \frac{\lambda_{d}}{d}\right) \Delta+A . R
$$

ProOF. Put

$$
f(s)=\sum_{n=1}^{\infty}\left(\sum_{\substack{d \backslash n \\ d<D}} \lambda_{d}\right) e\left(\frac{a}{q} n\right) n^{-s}
$$

which is absolutely convergent for $\operatorname{Re}(s)>1$, since $|f(s)| \leqq \zeta^{2}(\operatorname{Re}(s))$. On writing $n=d m, m=(d, q) l$ and $q=(d, q) r$, we eliminate $n$ and $m$. Then the conditions on $l$ and $r$ are

Thus, we have

$$
r \mid q, \quad(l, r)=1 \text {. }
$$




$$
\begin{aligned}
f(s)= & \sum_{d<D} \sum_{r \mid q} \sum_{b=1}^{r} * \sum_{\substack{l>0 \\
l \equiv b(r)}} \lambda_{d} e\left(\frac{a}{q} \frac{d q l}{r}\right)\left(\frac{d q l}{r}\right)^{-s} \\
= & \frac{1}{q^{s}} \sum_{r \mid q} \sum_{b=1}^{r} *\left(\sum_{d<D} \lambda_{d} e\left(\frac{a b d}{r}\right) d^{-s}\right) r^{s}\left(\sum_{\substack{l>0 \\
l \equiv b(r)}} l^{-s}\right) \\
& =\frac{1}{q^{s}} \sum_{r \mid q} \sum_{b=1}^{r} * D\left(s, \frac{b}{r}\right) \zeta\left(s, \frac{b}{r}\right), \text { say. }
\end{aligned}
$$

Hence $f(s)$ is regular, except for a simple pole at $s=1$. By Perron's formula [15, Anhang Satz 3.1.],

$$
u=\frac{1}{2 \pi i} \int_{c-i x}^{c+i x} f(s) \theta(s) d s+O\left((\log x)^{3}\right),
$$

where $c=1+(\log x)^{-1}$. Now,

$$
D\left(s, \frac{b}{r}\right)=\sum_{d \leq x / \Delta}+\sum_{x / \Delta<d<D}=D_{1}+D_{2}, \quad \text { say . }
$$

Let the corresponding expressions be $f_{1}$ and $f_{2}$, respectively. Put $\eta=\eta(x)=$ $c_{2}(\log x)^{-4 / 5}$. On moving the line of integration, we have

$$
\begin{aligned}
& u=\operatorname{Res}_{\boldsymbol{s}=1} f(s) \boldsymbol{\theta}(s) \\
& +\frac{1}{2 \pi i} \int_{1 / 2-i x}^{1 / 2+i x} f_{1}(s) \theta(s) d s+O\left(\int_{1 / 2 \pm i x}^{c \pm i x}\left|f_{1}(s) \theta(s)\right||d s|\right) \\
& +\frac{1}{2 \pi i} \int_{1-\eta-i x}^{1-\eta+i x} f_{2}(s) \theta(s) d s+O\left(\int_{1-\eta \pm i x}^{c \pm i x}\left|f_{2}(s) \theta(s)\right||d s|\right)+A . R . \\
& \text { (4.10) }=u_{0}+u_{1}+u_{2}+O\left(u_{3}\right)+A . R ., \text { say. }
\end{aligned}
$$

First,

$$
u_{0}=\operatorname{Res}_{s=1}=\frac{\Delta}{q} \sum_{r \mid q} \sum_{b=1}^{r} * D\left(1, \frac{b}{r}\right)=\Delta\left(\sum_{\substack{d<D \\ q \backslash d}} \frac{\lambda_{d}}{d}\right) .
$$

Since $\theta(s) \ll x^{\operatorname{Re}(s)-1}$ for $|\operatorname{Im}(s)|=x$, we have

$$
\begin{aligned}
u_{3} & \ll \sup _{\substack{1 / 2 \leq \sigma \leq c \\
j=1,2}}\left|f_{j}(\sigma \pm i x)\right| x^{\sigma-1} \\
& \ll \sup _{1 / 2 \leq \sigma \leq c} \sum_{r \mid q} \sum_{b=1}^{r} * D^{1-\sigma} \mathcal{L}\left|\zeta\left(\sigma \pm i x, \frac{b}{r}\right)\right| x^{\sigma-1} \\
& \ll \sup _{1 / 2 \leq \sigma \leq c} q^{2} \mathcal{L}^{2}\left(D x^{-2 / 3}\right)^{1-\sigma} \\
& \ll q^{2} x^{1 / 6},
\end{aligned}
$$

by Lemma 1. Hence, because of (4.1), $u_{3}$ is admissible.

We proceed to $u_{1}$. Lemma 1 yields that 


$$
\begin{aligned}
\left|u_{1}\right|_{2} & \ll \mathcal{L}^{2} \sup _{x / \Delta \leq U \leq x}\left(\frac{x}{U}\right)^{2} \sum_{r \mid q} \sum_{b=1}^{r} * \int_{1 / 2-i U}^{1 / 2+i U}\left|D_{1}\left(s, \frac{b}{r}\right) \zeta\left(s, \frac{b}{r}\right)\right|^{2}|d s| \\
& \ll \mathcal{L}^{2} \sup _{x / \Delta \leqq U \leqq x}\left(\frac{x}{U}\right)^{2} \sum_{r \mid q} \sum_{b=1}^{r} *\left(\sup _{\substack{\operatorname{Re}(s)=1 / 2 \\
\operatorname{Im}(s) \mid \leq U}}\left|\zeta\left(s, \frac{b}{r}\right)\right|^{2}\right) \int_{1 / 2-i U}^{1 / 2+i U}\left|D_{1}\left(s, \frac{b}{r}\right)\right|^{2}|d s| \\
& \ll \mathcal{L}^{5} \sup _{x / \Delta \leqq U \leqq x}\left(\frac{x}{U}\right)^{2} q U^{1 / 3}\left(U+\frac{x}{\Delta}\right) \\
& \ll \mathcal{L}^{5} \Delta^{2} q\left(\frac{x}{\Delta}\right)^{4 / 3} .
\end{aligned}
$$

Since $\Delta \geqq x^{1 / 4+\varepsilon^{\prime}}, u_{1}$ is admissible.

We turn now to $u_{2}$. As before, by Lemma 1, we have

$$
\begin{aligned}
\left|u_{2}\right|_{2} & \ll \mathcal{L}^{2} \sup _{x / \Delta \leqq U \leqq x} x^{1+2(1-\eta)} U^{-2} \sum_{r \mid q} \sum_{b=1}^{r} * \int_{1-\eta-i U}^{1-\eta+i U}\left|D_{2}\left(s, \frac{b}{r}\right) \zeta\left(s, \frac{b}{r}\right)\right|^{2}|d s| \\
& \ll q \mathcal{L}^{4} \exp \left(2 c_{1}(\log \log x)^{3}\right) x^{3-2 \eta} \sup _{\substack{x / \Delta \leq U \leq x \\
x / \Delta \leqq N \leqq D}} U^{-2}(U+N) N^{1-2(1-\eta)} \\
& \ll q \mathcal{L}^{4} \exp \left(2 c_{1}(\log \log x)^{3}\right) \Delta^{2} x\left(\frac{D}{x}\right)^{2 \eta} .
\end{aligned}
$$

Since $\eta=c_{2}(\log x)^{-4 / 5}, D \leqq x^{1-\varepsilon}$ and $q \leqq(\log x)^{B}$, we finally obtain

$$
\left|u_{2}\right|_{2} \ll \Delta^{2} x \exp \left(-\varepsilon c_{2}(\log x)^{-1 / 6}\right) .
$$

Thus, $u_{2}$ is also admissible.

In conjunction with (4.10)-(4.14), we have

as required.

$$
u=u_{0}+A . R .
$$

\section{Auxiliary results.}

In this section we provide for the proof of Lemmas 16 and 17 in the next section. For real numbers $a, b$ and $c, a \leqq b$, we set

$$
\begin{aligned}
\Phi(a, b ; c) & =\psi(b-c)-\psi(a-c) \\
& =[b-c]-[a-c]-(b-a) .
\end{aligned}
$$

Let $\alpha, \beta, \gamma$ and $\delta$ be arbitrary sequences with modulus $\leqq 1$. Moreover, let 1 denote the arithmetical function $1(n) \equiv 1$ or $\equiv \log n$. We consider the following linear forms involving $\Phi$.

$$
\begin{aligned}
& F_{1}=F_{1}(K, D, M, N ; x, s, t)
\end{aligned}
$$

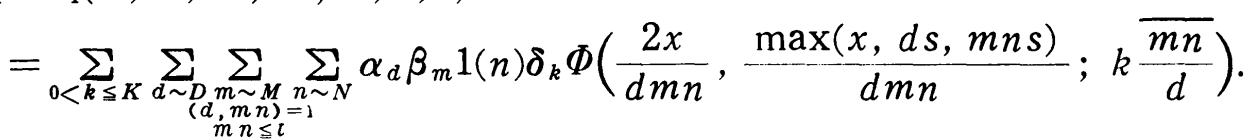




$$
\begin{aligned}
& F_{2}=F_{2}(K, D, M, N ; x, s, t)
\end{aligned}
$$

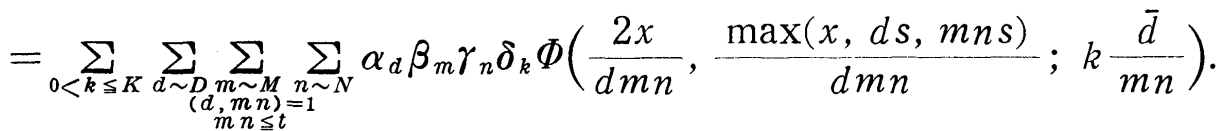

Here, $1 \ll K, D, M, N \ll x, M N \leqq t$ and $s \leqq 2 x / D, 2 x / M N$.

Our aim is to prove $F \ll K x^{1-\varepsilon}, \varepsilon>0$. Since $F \ll K D M N$ trivially, we assume

$$
D M N>x^{1-2 \varepsilon} \text {. }
$$

LEMMA 13. For any $\varepsilon>0$, if $M N, D M \leqq x^{2 / 3-4 \varepsilon}$ then

$$
F_{1} \ll K x^{1-\varepsilon} .
$$

Proof. We appeal to the Erdös-Turán inequality [8, Lemme 2], which runs as follows. For arbitrary real numbers $x_{n}$ and $H>2$,

$$
\left|\sum_{n \sim N} \psi\left(x_{n}\right)\right| \ll \frac{N}{H}+\sum_{0<h \leqq H} \frac{1}{h}\left|\sum_{n \sim N} e\left(h x_{n}\right)\right| .
$$

Now, by partial summation, we have

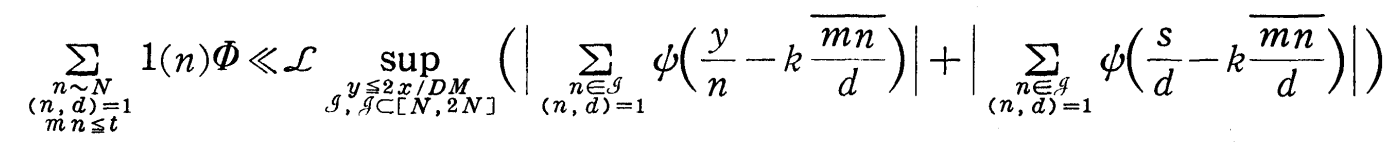

$$
\begin{aligned}
& \ll \mathcal{L} \sup _{\substack{y \leq 2 x, D M \\
g \subset[N, 2 N]}}\left(\frac{N}{H}+\sum_{0<h \leqq H} \frac{1}{h}\left|\sum_{\substack{n \in \mathcal{G} \\
(n, d)=1}} e\left(\frac{h y}{n}\right) e\left(-h k \frac{\overline{m n}}{d}\right)\right|\right) .
\end{aligned}
$$

It follows from the argument of $[11$, p. 37] that

$$
\begin{aligned}
F_{1} & \ll \mathcal{L} \frac{K D M N}{H}+\mathcal{L}^{2} \sum_{0<h \leqq H} \frac{1}{h} x^{\varepsilon}\left(1+\frac{H x}{D M N}\right) \tau(h) K M\left(D^{3 / 2}+D^{1 / 2} N\right) \\
& \ll \mathcal{L} \frac{K D M N}{H}+\mathcal{L}^{4} x^{\varepsilon}\left(1+\frac{H x}{D M N}\right) K x^{1-6 \varepsilon} .
\end{aligned}
$$

On taking $H=D M N / x^{1-3 \varepsilon}>2$, because of (5.1), we obtain Lemma 13.

LEMMA 14. For any $\varepsilon>0$, if $D, M N \leqq x^{8 / 15-4 \varepsilon}$ and $N \geqq x^{1 / 15}$ then

$$
F_{2} \ll K x^{1-\varepsilon} .
$$

PRoOF. We follow the argument of [11] with a minor modification. We use a well known Fourier expansion. For $H>2$,

$$
\begin{aligned}
\Phi(a, b ; c)= & \sum_{0<|h| \leqq H} \frac{e(h b)-e(h a)}{2 \pi i h} e(-h c) \\
& +O(\min (1,1 / H\|b-c\|)+\min (1,1 / H\|a-c\|)) .
\end{aligned}
$$


Also,

$$
\min (1,1 / H\|x\|)=\sum_{h \in Z} A_{h} e(h x)
$$

where

$$
\left|A_{h}\right| \ll \min \left(\frac{\log H}{H}, \frac{H}{h^{2}}\right)
$$

Now, $\Phi$ in $F_{2}$ is expressed as a sum of the main part of Fourier series, say $\Phi^{\prime}$, and the tail part, say $\Phi^{\prime \prime}$. On putting the resulting linear forms to $F_{2}^{\prime}$ and $F_{2}^{\prime \prime}$, we may write

$$
F_{2}=F_{2}^{\prime}+F_{2}^{\prime \prime} .
$$

First we consider $F_{2}^{\prime \prime}$. (5.3) yields that

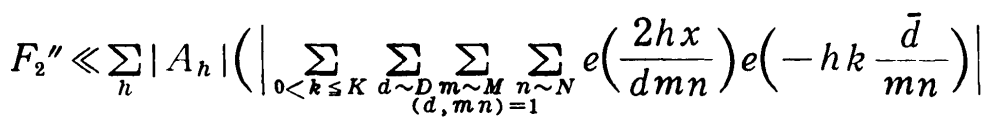

$$
\begin{aligned}
& \left.+\left|\sum_{0<k \leq K} \sum_{\substack{d \sim D \\
(d, m \sim M}} \sum_{\substack{m \sim 1 \\
(d) N}} e\left(\frac{h \max (x, d s, m n s)}{d m n}\right) e\left(-h k \frac{\bar{d}}{m n}\right)\right|\right) \\
& =\sum_{h}\left|A_{h}\right| S(h) \text {, say. }
\end{aligned}
$$

Summing by parts, we see

$$
S(h) \ll \sum_{0<k \leq K} \sum_{m \sim M} \sum_{n \sim N}\left(1+\frac{h x}{D M N}\right) \sup _{g \subset[D, 2 D]}\left|\sum_{\substack{d \in \mathcal{G} \\(\alpha, m n)=1}} e\left(h k \frac{\bar{d}}{m n}\right)\right| .
$$

By the same argument as that in $[11,(17)(18)]$ with $q=1$, we then obtain

$$
F_{2}^{\prime \prime} \ll K x^{1-2 \varepsilon}+\mathcal{L}^{3} x^{4 \varepsilon} K\left\{(M N)^{3 / 2}+D(M N)^{1 / 2}\right\} .
$$

Here we have taken $H=D M N x^{3 \varepsilon-1}>2$, because of $(5.1)$. Since $D, M N<x^{2 / 3-4 \varepsilon}$, we have

$$
F_{2}^{\prime \prime} \ll K x^{1-\varepsilon} .
$$

We turn now to $F_{2}^{\prime}$. By (5.2),

$$
\begin{aligned}
& F_{2}^{\prime}=\sum_{0<k \leq K} \sum_{\substack{d \sim D \\
d \sim n \sim N \\
(d, m n)=1 \\
m n \leq t}} \sum_{\substack{n \\
m \leq t}} \alpha_{d} \beta_{m} \gamma_{n} \delta_{k} \\
& \times \sum_{0<|h| \leq H} \int_{\max (x, d s, m n s) g / d m n}^{2 x g / d m n} e\left(\frac{h}{g} y\right) \frac{d y}{g} \cdot e\left(-h k \frac{\bar{d}}{m n}\right) \text {, }
\end{aligned}
$$

where $g$ is arbitrary. After some elementary rearrengement, we reach the inequality

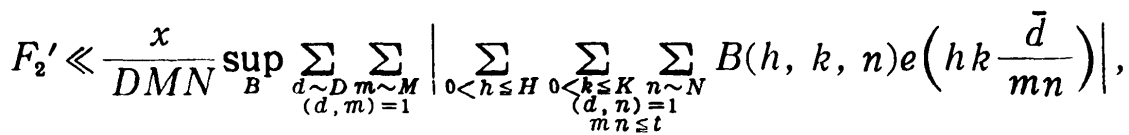


where the supremum is over all arithmetical functions $B$ with $|B| \leqq 1$. To deal with the condition $m n \leqq t$, we use a lemma in Fourier analysis [1, Lemma 2.2.], which runs as follows. Let $a_{n}$ be arbitrary finite sequence. If $X \leqq Y$ then

$$
\left|\sum_{n \leq X} a_{n}\right| \leqq \int_{-\infty}^{\infty} f(\theta, Y)\left|\sum_{n} a_{n} e(\theta n)\right| d \theta .
$$

Here, the kernel $f \geqq 0$ satisfies

$$
\int_{-\infty}^{\infty} f(\theta, Y) d \theta \ll \log Y
$$

Now, applying this lemma with $Y=t / M$, we may remove the condition $m n \leqq t$ in (5.4) with the cost of $(\log x)$ time. Thus, the method in $[11, \mathrm{p} .38$, 39] is applicable. We therefore have

$$
F_{2}^{\prime} \ll \mathcal{L} x^{3 \varepsilon}\left\{(K D M)^{1 / 2} x^{1 / 2-\varepsilon}+K x^{5 \varepsilon / 2}\left(D^{1 / 2} M^{5 / 4} N^{3 / 2}+D M^{3 / 4} N^{1 / 2}\right)\right\} .
$$

Since $D M<x^{8 / 15-4 \varepsilon} \cdot x^{7 / 16-4 \varepsilon}=x^{1-8 \varepsilon}$, the above first term is

$$
\begin{aligned}
& \ll \mathcal{L} x^{3 \varepsilon} K^{1 / 2} x^{1-\delta \varepsilon} \\
& \ll K x^{1-\varepsilon} .
\end{aligned}
$$

We may assume $M \geqq N$, because of the symmetry of $F_{2}$, so that $M \geqq(M N)^{1 / 2}$. Hence, the second term is

$$
\begin{aligned}
& \ll K x^{6 \varepsilon}\left\{D^{1 / 2}(M N)^{3 / 2-1 / 8}+D(M N)^{1 / 2} M^{1 / 4}\right\} \\
& \ll K x^{6 \varepsilon}\left\{\left(x^{8 / 15-4 \varepsilon}\right)^{15 / 8}+\left(x^{8 / 15-4 \varepsilon}\right)^{3 / 2}\left(x^{7 / 15-4 \varepsilon}\right)^{1 / 4}\right\} \\
& \ll K x^{1-\varepsilon} .
\end{aligned}
$$

We thus obtain Lemma 14.

\section{Minor arc, preliminaries.}

In this section we provide for the final section. We re-present the method of [10] with a few simplifications. Let $a(n), b(n)$ and $c(n)$ be arbitrary arithmetical functions which are bounded by

$$
\tau_{c_{7}}(n)(\log n)^{c_{8}} \text {. }
$$

Also, $1(n) \equiv 1$ or $\equiv \log n$. Furthermore, we put

$$
\begin{gathered}
j \mathrm{I}(h ; s)=\sum_{\substack{h=d l \\
l \geq s}} a(d), \\
j \Pi(h ; D, V)=\sum_{\substack{h=l m n \\
m n \leq s \\
m \leq V}} 1(l) a(m) 1(n),
\end{gathered}
$$




$$
\begin{gathered}
j \mathrm{III}(h ; D, U)=\sum_{\substack{n=l m n \\
m n \leq D \\
m, n \geq U}} 1(l) a(m) b(n), \\
j \operatorname{IV}(h ; U, V, \mathcal{G})=\sum_{\substack{h=m n k \\
V<m \leq U \\
m \in \in \mathcal{M} \\
k \in \mathcal{G}}} a(m) b(n) c(k) .
\end{gathered}
$$

Here, $s, D, U, V$ 's are parameters and $g$ is an interval. We are devoted to estimating the mean value of trigonometrical sums

$$
\begin{gathered}
J \boldsymbol{i}(*)=J \boldsymbol{i}(\alpha, x, \Delta ; *)=\Delta^{2} \int_{-1 / 2 \Delta}^{1 / 2 \Delta}\left|\sum_{x<h \leq 2 x} j \boldsymbol{i}(h ; *) e((\alpha+\beta) h)\right|^{2} d \beta \\
(i=\mathrm{I}, \mathrm{II}, \mathrm{III}, \mathrm{IV})
\end{gathered}
$$

under the assumption

$$
\left|\alpha-\frac{a}{q}\right| \leqq \frac{1}{q^{2}} \quad \text { with } \quad(a, q)=1 \quad \text { and } \quad 1<q<\Delta<x / 2 .
$$

LEMMA 15 . For any $\varepsilon>0$,

$$
J \mathrm{I}(s) \ll \Delta x \mathcal{L}^{F}\left\{\Delta q^{-1 / 2}+(q \Delta)^{1 / 2}\right\}+\Delta^{2}\left(\frac{x}{s}\right)^{2} \mathcal{L}^{F}+\Delta^{3} x^{\varepsilon} .
$$

Proof. On writing $w_{n}=j \mathrm{I}(n ; s)$, Lemma 8 yields that

$$
\begin{aligned}
J I & \ll \Delta \sum_{n \sim x}\left|w_{n}\right|^{2}+\Delta \sup _{t \leq \Delta}\left|\sum_{0<r \leq t} e(\alpha r) \sum_{n \sim x} \bar{w}_{n} w_{n+r}\right|+\Delta^{3}\left(\sup \left|w_{n}\right|\right)^{2} \\
& \ll \Delta x \mathcal{L}^{F}+\Delta \sup |\Sigma|+\Delta^{3} x^{\varepsilon},
\end{aligned}
$$

where

$$
\Sigma=\sum_{0<r \leqq t} e(\alpha r) \sum_{d_{1}} \sum_{d_{2}} a\left(d_{1}\right) a\left(d_{2}\right) \#\left\{\begin{array}{c}
\max \left(x, d_{1} s, d_{2} s\right)<n \leqq 2 x \\
n: \begin{array}{l}
n \equiv 0\left(\bmod d_{1}\right) \\
n \equiv r\left(\bmod d_{2}\right)
\end{array}
\end{array}\right\}+O\left(\Delta^{2} x^{\varepsilon}\right) .
$$

The above simultaneous congruences are soluble if and only if $\left(d_{1}, d_{2}\right) \mid r$. We write $d_{i}^{*}=d_{i} /\left(d_{1}, d_{2}\right)$ and $r^{*}=r /\left(d_{1}, d_{2}\right)$. We then have, with the notation in section 5 , that

$$
\begin{aligned}
\#\{n\} & =\#\left\{l: \frac{\max \left(x, d_{1} s, d_{2} s\right)}{\left[d_{1}, d_{2}\right]}<r^{*} \frac{\overline{d_{1}^{*}}}{d_{2}^{*}}+l \leqq \frac{2 x}{\left[d_{1}, d_{2}\right]}\right\} \\
& =\frac{2 x-\max \left(x, d_{1} s, d_{2} s\right)}{\left[d_{1}, d_{2}\right]}+\Phi\left(\frac{2 x}{\left[d_{1}, d_{2}\right]}, \frac{\max \left(x, d_{1} s, d_{2} s\right)}{\left[d_{1}, d_{2}\right]} ; r^{*} \frac{\overline{d_{1}^{*}}}{d_{2}^{*}}\right) \\
& =\Phi_{0}+\Phi, \text { say. }
\end{aligned}
$$

If $d_{1}$ or $d_{2}>2 x / s$ then $\#\{n\}=0$. Hence

$$
\begin{aligned}
\Sigma & =\sum_{0<r \leqslant t} e(\alpha r) \sum_{\substack{\alpha_{1}, d_{2} \leq 2 x / s \\
\left(d_{1}, d_{2}\right) \mid r}} a\left(d_{1}\right) a\left(d_{2}\right)\left(\Phi_{0}+\Phi\right)+O\left(\Delta^{2} x^{\varepsilon}\right) \\
& =\Sigma_{0}+\Sigma_{1}+O\left(\Delta^{2} x^{\varepsilon}\right), \text { say. }
\end{aligned}
$$


It follows from [15, Kap. IV Lemma 6.3.] that

$$
\begin{aligned}
\Sigma_{0} & \ll x \sum_{\substack{d_{1}, d_{2} \leq 2, x / s \\
\left(d_{1}, d_{2}\right) \leq t}} \frac{\left|a\left(d_{1}\right) a\left(d_{2}\right)\right|}{\left[d_{1}, d_{2}\right]}\left|\sum_{\substack{0<r \leq t \\
\left(d_{1}, d_{2}\right) \mid r}} e(\alpha r)\right| \\
& \ll x \sum_{0<\delta \leq \Delta}\left(\delta \sum_{\substack{0 \leq 2 x / s \\
\delta ! d}} \frac{|a(d)|}{d}\right)^{2} \delta^{-1} \min \left(\frac{\Delta}{\delta}, \frac{1}{\|\alpha \delta\|}\right) \\
& \ll x\left(\sum_{\delta \leqq \Delta}\left(\delta \sum_{\substack{d \leq 2 x \\
\delta \mid d}} \frac{|a(d)|}{d}\right)^{4} \delta^{-2}\right)^{1 / 2}\left(\sum_{0<\delta \leq \Delta} \frac{\Delta}{\delta} \min \left(\frac{\Delta}{\delta}, \frac{1}{\|\alpha \delta\|}\right)\right)^{1 / 2} \\
& \ll x \mathcal{L}^{F} \Delta^{1 / 2}\left(\frac{\Delta}{q}+q\right)^{1 / 2} .
\end{aligned}
$$

Also, since $\Phi \ll 1$,

$$
\begin{aligned}
& \Sigma_{1} \ll \Delta\left(\sum_{d \leqq 2 x / s}|a(d)|\right)^{2} \\
& \ll \Delta\left(\frac{x}{s}\right)^{2} \mathcal{L}^{F} .
\end{aligned}
$$

In conjunction with (6.2)-(6.5) we obtain Lemma 15 .

LEMMA 16 . For any $\varepsilon>0$, if $D V \leqq x^{2 / 3-8 \varepsilon}$ then

$$
J \mathrm{II}(D, V) \ll \Delta x \mathcal{L}^{F}\left\{\Delta q^{-1 / 2}+(q \Delta)^{1 / 2}\right\}+\Delta^{2} x^{1-\varepsilon}+\Delta^{3} x^{\varepsilon} .
$$

PRoOF. We have, by the same argument as before, that

where

$$
\begin{aligned}
J \Pi \ll \Delta x \mathcal{L}^{F}\left\{\Delta q^{-1 / 2}+(q \Delta)^{1 / 2}\right\}+\Delta^{3} x^{\varepsilon} \\
+\Delta^{2} \mathcal{L}^{F} x^{1-2 \varepsilon}+\sup _{\substack{t \leq \Delta \\
s \leq x^{1 / 2+\varepsilon}}}\left|\Sigma_{8, t}\right|,
\end{aligned}
$$

$$
\begin{aligned}
\Sigma= & \sum_{0<r \leqq t} e(\alpha r)_{d_{1}, d_{2} \leq \min (2 x</ s, D)} w\left(d_{1}\right) w\left(d_{2}\right) \\
\left(d_{1}, d_{2}\right) \mid r & \\
& \times \Phi\left(\frac{2 x}{\left[d_{1}, d_{2}\right]}, \frac{\max \left(x, d_{1} s, d_{2} s\right)}{\left[d_{1}, d_{2}\right]} ; r^{*} \frac{\overline{d_{1}^{*}}}{d_{2}^{*}}\right)
\end{aligned}
$$

with

$$
w(d)=\sum_{\substack{d=m^{\prime} n^{\prime} \\ m^{\prime} \leqq V}} a\left(m^{\prime}\right) 1\left(n^{\prime}\right)
$$

Write

$$
\begin{aligned}
& \left(d_{1}, d_{2}\right)=\delta, \\
& d_{1}=\delta m n, \quad \delta=e f, \quad m^{\prime}=e m, \quad n^{\prime}=f n, \\
& d_{2}=\delta d, \\
& r^{*}=k .
\end{aligned}
$$

We then find 


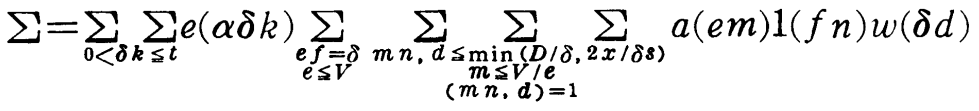

$$
\begin{aligned}
& \times \Phi\left(\frac{2 x / \delta}{m n d}, \frac{\max (x / \delta, m n s, d s)}{m n d} ; k \frac{\overline{m n}}{d}\right) .
\end{aligned}
$$

On breaking up the ranges for $m, n$ and $d$ into $O\left((\log x)^{3}\right)$ intervals of the shape $\left[2^{i}, 2^{i+1}\right]$, we conclude that

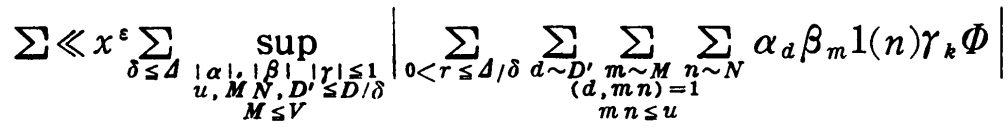

$$
\begin{aligned}
& =x^{\varepsilon} \sum_{\delta \leq \Delta} \sup _{D^{\prime}, M N \leq D / \delta}\left|F_{1}\left(\frac{\Delta}{\delta}, D^{\prime}, M, N ; \frac{x}{\delta}, s, u\right)\right|,
\end{aligned}
$$

where $F_{1}$ is defined in section 5 . Since

$$
\frac{D V}{\delta}, \frac{D}{\delta} \leqq\left(\frac{x}{\delta}\right)^{2 / 3-8 \varepsilon}
$$

Lemma 13 yields

$$
\begin{aligned}
\Sigma & \ll x^{\varepsilon} \sum_{\delta \leq \Delta}\left(\frac{\Delta}{\delta}\right)\left(\frac{x}{\delta}\right)^{1-2 \varepsilon} \\
& \ll \Delta x^{1-\varepsilon} .
\end{aligned}
$$

Combining this with (6.6) we obtain Lemma 16.

LEMMA 17. For any $\varepsilon>0$, if $D \leqq x^{8 / 16-12 \varepsilon}$ and $U \geqq x^{1 / 5}$ then

$$
J \mathrm{III}(D, U) \ll \Delta x \mathcal{L}^{F}\left\{\Delta q^{-1 / 2}+(q \Delta)^{1 / 2}\right\}+\Delta^{2} x^{1-\varepsilon}+\Delta^{3} x^{\varepsilon} .
$$

Proof. It suffices to show

$$
\sum_{\delta \leq \Delta} \sup _{\substack{u, D^{\prime}, M N \leq D / \delta \\ N>\max (U / \delta, 1) \\ \delta \leq x^{1 / 2+\varepsilon}}}\left|F_{2}\left(\frac{\Delta}{\delta}, D^{\prime}, M, N ; \frac{x}{\delta}, s, u\right)\right| \ll \Delta x^{1-2 \varepsilon} .
$$

When $\delta>D^{2} x^{2 \varepsilon-1}$, we use the trivial bound

$$
F_{2} \ll \frac{\Delta}{\delta}\left(\frac{D}{\delta}\right)^{2} .
$$

Otherwise, we make use of Lemma 14. Since

$$
\frac{D}{\delta} \leqq\left(\frac{x}{\delta}\right)^{8 / 15-12 \varepsilon} \text { and } \frac{U}{\delta} \geqq\left(\frac{x}{\delta}\right)^{1 / 15} \text {, }
$$

Lemma 14 yields

$$
F_{2} \ll\left(\frac{\Delta}{\delta}\right) x^{1-3 \varepsilon}
$$


We therefore have

$$
\begin{aligned}
\sum_{\delta \leqq \Delta} \sup \left|F_{2}\right| & \ll \sum_{\delta \leqq D^{2} x^{2 \varepsilon-1}}\left(\frac{\Delta}{\delta}\right) x^{1-3 \varepsilon}+\sum_{D^{2} x^{2 \varepsilon-1}<\delta \leqq \Delta}\left(\frac{\Delta}{\delta}\right)\left(\frac{D}{\delta}\right)^{2} \\
& \ll \Delta x^{1-2 \varepsilon},
\end{aligned}
$$

as required.

LEMMA 18.

$$
J \operatorname{IV}(U, V, g) \ll \Delta x \mathcal{L}^{F}\left(U+\frac{\Delta}{q}+\frac{\Delta}{V}+q\right) .
$$

Proof. We may assume $U \leqq \Delta$, for otherwise Lemma is trivial. We split the ranges for $m, n$ and $k$ into $O\left((\log x)^{3}\right)$ intervals of the form $(M, 2 M]$, $(N, 2 N]$ and $(K, 2 K]$, so that

$$
x \ll M N K \ll x \text { and } V \leqq M \leqq U .
$$

Using Cauchy's inequality twice, we have

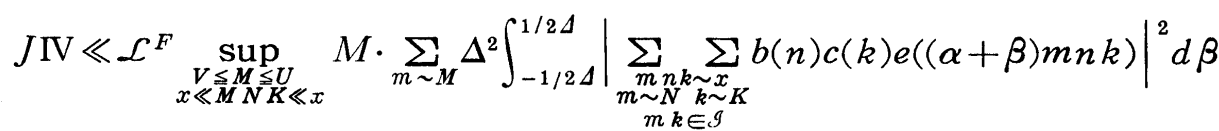

$$
\begin{aligned}
& \ll \mathcal{L}^{F} \sup M \cdot J \text {, say. }
\end{aligned}
$$

By Lemma 8,

$$
\begin{aligned}
& J \ll \sum_{m \sim M} \Delta \sum_{N K<l \leqq 4 N K}\left(\sum_{l=n k}|b(n) c(k)|\right)^{2} \\
& +\sum_{0<r \leq \Delta} \sum_{\substack{r=n k, n^{\prime} k^{\prime} \\
n, n, k^{\prime} \sim N \\
k^{\prime}, k^{\prime}}}\left|b(n) c(k) b\left(n^{\prime}\right) c\left(k^{\prime}\right)\right| \cdot\left|\sum_{\substack{m \sim m \\
k m, k^{\prime} m \in \mathcal{G}}} \max (0, \Delta-m r) e(\alpha m r)\right| \\
& \ll \Delta \mathcal{L}^{F} M N K \\
& +N K \mathcal{L}^{F} \sum_{0<r \leqq \Delta} \sup _{\mathcal{G}}\left|\sum_{\substack{m \in \mathcal{G} \\
m \geqq M}} \max (0, \Delta-m r) e(\alpha m r)\right| \\
& \ll \Delta x \mathcal{L}^{F}+N K \mathcal{L}^{F} \sum_{0<r \leqq \Delta, M} \sup _{g}\left|\sum_{\substack{m \in g \\
m \leq M \\
0<m r}}(\Delta-m r) e(\alpha m r)\right| \\
& \text { (6.8) }=\Delta x \mathcal{L}^{F}+N K \mathcal{L}^{F} \cdot \mathscr{I}, \text { say. }
\end{aligned}
$$

Summing by parts, we have

$$
\begin{aligned}
\mathscr{I} & \ll \sum_{0<r \leq \Delta / M} \Delta \min \left(\frac{\Delta}{r}, \frac{1}{\|\alpha r\|}\right) \\
& \ll \Delta\left(\frac{\Delta}{q}+\frac{\Delta}{M}+q\right),
\end{aligned}
$$

by [15, Kap. VI Lemma 6.3.]. Combining this with (6.7) and (6.8), we finally 
obtain

$$
\begin{aligned}
J \mathrm{IV} & \ll \mathcal{L}^{F} \sup _{\substack{V \leq M \leq U \\
x \ll M K}} M\left\{\Delta x+N K \cdot \Delta\left(\frac{\Delta}{q}+\frac{\Delta}{M}+q\right)\right\} \\
& \ll \Delta x \mathcal{L}^{F}\left(U+\frac{\Delta}{q}+\frac{\Delta}{V}+q\right) .
\end{aligned}
$$

\section{Minor arc.}

In this section we prove Lemmas $10^{\dagger}$ and $12^{\dagger}$ below, which cover Lemmas 10 and 12 , respectively. Throughout this section we assume that

$$
\left|\alpha-\frac{a}{q}\right| \leqq \frac{1}{q^{2}} \quad \text { with } \quad(a, q)=1, \quad \text { and } \quad 1<\Delta<x^{2 / 3} .
$$

Lemma $10^{\dagger}$. Put

$$
\mathscr{I}=\mathscr{I}(\alpha, x, \Delta, N)=\left.\Delta^{2} \int_{-1 / 2 \Delta}^{1 / 2 \Delta}||_{\substack{x<m n \leq 2 x \\ \mathcal{J}<n \leq N}} \Lambda(m) \Lambda(n) e((\alpha+\beta) m n)\right|^{2} d \beta .
$$

For any $E, \varepsilon>0$, if $N \leqq x^{8 / 15-\varepsilon}$ then

$$
\mathscr{I} \ll \Delta x \mathcal{L}^{F}\left\{x^{1 / 4}+\Delta q^{-1 / 2}+(q \Delta)^{1 / 2}\right\}+\Delta^{2} x \mathcal{L}^{-E},
$$

where the implied constant depends only on $E$ and $\varepsilon$.

Proof. We may assume $q \leqq \Delta$, for otherwise Lemma is trivial. For $x<$ $h \leqq 2 x$, put

$$
\rho(h)=\sum_{\substack{h=m n \\ n \in \mathcal{I}}} A(m) \Lambda(n), \quad g=(\sqrt{x}, N] .
$$

We shall decompose $\rho$ into $O(1)$ sums of the $j i$-type weight, which is defined in section 6. In order to apply Lemma 6, we introduce the prameters $u, v$ and $w$ such that

$$
\begin{aligned}
& u=2 x^{1 / 4} \\
& v=N x^{-1 / 4} \\
& w=x^{\xi}, \quad \varepsilon=20 \xi .
\end{aligned}
$$

Then, for $x<m n \leqq 2 x$ and $n \in \mathcal{G}$,

$$
u<\frac{x}{N}<m \leqq 2 \sqrt{x}<u^{2} \text { and } v<\sqrt{x}<n \leqq N<u v .
$$

Lemma 6 yields that, for $x<h \leqq 2 x$, 


$$
\rho(h)=\sum_{\substack{h=m m^{\prime} n n^{\prime} \\ n n^{\prime} \in g^{\prime} \\ m, \backslash u \\ n^{\prime}>v}}\left(\sum_{\substack{d \uparrow m \\ d \leq u}} \mu(d)\right) \Lambda\left(m^{\prime}\right)\left(\sum_{\substack{e \nmid n \\ e \leqq u}} \mu(e)\right) \Lambda\left(n^{\prime}\right) .
$$

We now divide the range of variables in $\rho$ as follows.

$$
\begin{aligned}
& d \in(0, u]=H_{2} \cup H_{3}, \\
& e \in(0, u]=H_{2} \cup H_{3}, \\
& m^{\prime} \in(u, \infty)=H_{1} \backslash H_{3}, \\
& n^{\prime} \in(v, \infty)=H_{1} \backslash\left(H_{3} \cup H_{4}\right) .
\end{aligned}
$$

Here, $H_{1}=(w, \infty), H_{2}=(0, w], H_{3}=(w, u]$ and $H_{4}=(u, v]$. Thus, we may write

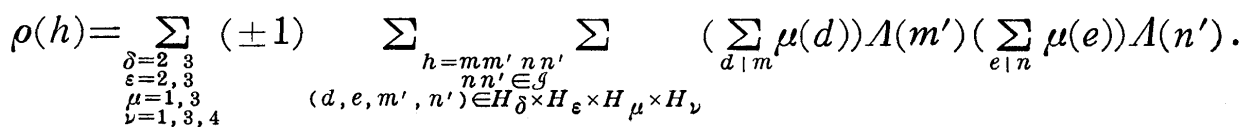

Let $\rho_{2}$ and $\rho_{3}$ be the sums corresponding to

$$
\begin{array}{lll}
\delta=2 & & \delta=2 \\
\varepsilon=2 & & \varepsilon=2,3 \\
\mu=1 & \text { and } & \\
\nu=1 & & \nu=1 \\
& \nu=4
\end{array}
$$

respectively. Moreover, let $\rho_{4}$ denote the remaining sums, so that

$$
\rho(h)=\rho_{2}(h)-\rho_{3}(h)+\rho_{4}(h) .
$$

We first consider $\rho_{4}$. We easily see that at least one of $\delta, \varepsilon, \mu$ or $\nu$ must be 3. Namely, at least one of $d, e, m^{\prime}$ or $n^{\prime}$ lies in the interval $(w, u]$. We therefore have that $\rho_{4}$ is splitted into 21 sums of the $j \operatorname{IV}(h ; u, w, g)$-type sum, with $g=g$ or $(0,2 x]$.

We proceed to $\rho_{2}$. On writing $m m^{\prime}=d f$ and $n n^{\prime}=e g$, we have

$$
\begin{aligned}
& \rho_{2}(h)=\sum_{\substack{h=m m m^{\prime} \\
n n^{\prime}, \sum^{\prime} n^{\prime} \\
m, m^{\prime}, \mathcal{g}^{\prime}}}\left(\sum_{\substack{d \uparrow m \\
d \leqq w^{\prime}}} \mu(d)\right) \Lambda\left(m^{\prime}\right)\left(\sum_{\substack{e \backslash n \\
e \leqq w}} \mu(e)\right) \Lambda\left(n^{\prime}\right) \\
& =\sum_{n=d} \sum_{\substack{f e g \\
e g \in \mathcal{g}}} \sum_{d \leq w} \mu(d)\left(\sum_{\substack{m^{\prime}, f \\
m^{\prime}>w}} \Lambda\left(m^{\prime}\right)\right) \sum_{e \leqq w} \mu(e)\left(\sum_{\substack{n, 1 g \\
n,>w}} \Lambda\left(n^{\prime}\right)\right)
\end{aligned}
$$

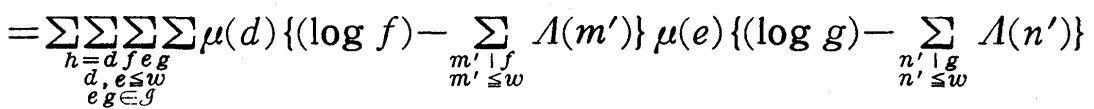

Thus, $\rho_{2}(h)$ is divided into 4 sums of the $j \Pi\left(h ; w^{2} N, w^{4}\right)$-type sum.

We turn now to $\rho_{3}$. 


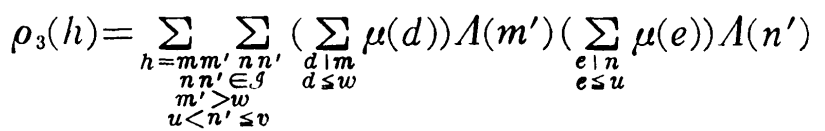

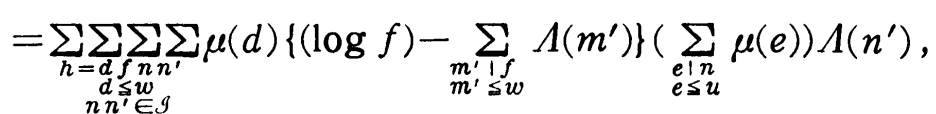

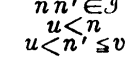

since $n n^{\prime} \in \mathcal{G}$ and $u<n^{\prime} \leqq v$ imply $n>1$. Hence, $\rho_{3}(h)$ is a sum of two $j \amalg(h$; $w^{2} N, u$ )-type sums.

We therefore conclude that

$$
\mathscr{I} \ll J \Pi\left(w^{2} N, w^{4}\right)+J \mathbb{\Pi}\left(w^{2} N, u\right)+J \operatorname{IV}(u, w, g) .
$$

We note that

$$
\begin{aligned}
& w^{6} N \leqq x^{8 / 15-\varepsilon+12 \xi} \leqq x^{2 / 3-8 \xi}, \\
& w^{2} N \leqq x^{8 / 15-\varepsilon+4 \xi} \leqq x^{8 / 15-12 \xi}, \\
& u=2 x^{1 / 4} \geqq x^{1 / 5} .
\end{aligned}
$$

Thus, Lemmas 16, 17 and 18 yield

$$
\begin{aligned}
\mathscr{I} & \ll \Delta x \mathcal{L}^{F}\left\{\Delta q^{-1 / 2}+(q \Delta)^{1 / 2}\right\}+\Delta^{2} x^{1-\xi}+\Delta x \mathcal{L}^{F}\left(u+\frac{\Delta}{q}+\frac{\Delta}{w}+q\right) \\
& \ll \Delta x \mathcal{L}^{F}\left\{x^{1 / 4}+\Delta q^{-1 / 2}+(q \Delta)^{1 / 2}\right\}+\Delta^{2} x^{1-\xi},
\end{aligned}
$$

as required.

LEMMA $12^{\dagger}$. Let $\left(\lambda_{d}\right)=\left(\lambda_{d}(D, z)\right)$ be the weights introduced in Lemma 5. Put

$$
\mathcal{S}=\mathcal{S}(\alpha, x, \Delta, D, z)=\Delta^{2} \int_{-1 / 2 \Delta}^{1 / 2 \Delta}\left|\sum_{x<n \leq 2 x}\left(\sum_{d \mid n} \lambda_{d}\right) e((\alpha+\beta) n)\right|^{2} d \beta .
$$

For any $E, \varepsilon>0$, if $4<z^{2} \leqq D \leqq x^{1-\varepsilon}$ then

$$
\mathcal{S} \ll \Delta x \mathcal{L}^{F}\left\{x^{1 / 4}+\Delta q^{-1 / 2}+(q \Delta)^{1 / 2}\right\}+\Delta^{2} x \mathcal{L}^{-E},
$$

where the implied constant depends only on $E$ and $\varepsilon$.

Proof. We may assume $q \leqq \Delta$. By Lemma 5, we have

Here,

$$
\mathcal{S} \ll J \mathrm{I}\left(x / z_{1} z\right)+\mathcal{L}^{2} J \operatorname{IV}\left(D^{1 / 4} z_{9}, z_{2},(0,2 x]\right) .
$$

$$
z_{i}=\exp \left((\log D)(\log \log D)^{-i / 10}\right) .
$$

Lemmas 15 and 18 yield that

$$
\begin{gathered}
\mathcal{S} \ll \Delta x \mathcal{L}^{F}\left\{\Delta q^{-1 / 2}+(q \Delta)^{1 / 2}\right\}+\Delta\left(z_{1} z\right)^{2} \mathcal{L}^{F} \\
+\Delta x \mathcal{L}^{F}\left(D^{1 / 4} z_{9}+\frac{\Delta}{q}+\frac{\Delta}{z_{2}}+q\right)
\end{gathered}
$$




$$
\begin{aligned}
& \ll \Delta x \mathcal{L}^{F}\left\{D^{1 / 4} z_{9}+\Delta q^{-1 / 2}+(q \Delta)^{1 / 2}\right\}+\Delta^{2} \mathcal{L}^{F} z_{1}{ }^{2} D+\Delta^{2} x \mathcal{L}^{F} z_{2}{ }^{-1} \\
& \ll \Delta x \mathcal{L}^{F}\left\{x^{1 / 4}+\Delta q^{-1 / 2}+(q \Delta)^{1 / 2}\right\}+\Delta^{2} x \mathcal{L}^{-E},
\end{aligned}
$$

for any $E>0$, as required.

This completes our proof of Theorem.

\section{References}

[1] Bombieri, E. and Iwaniec, H., On the order of $\zeta((1 / 2)+i t)$. Ann. Scuola Norm. Sup. Pisa 13 (1986), 449-472.

[2] Chen, J.-r., The exceptional set of Goldbach number, Sci. Sinica 26 (1983), 714-731.

[3] Gallagher, P.X., A large sieve density estimate near $\sigma=1$, Invent. Math. 11 (1970), 329-339.

[4] Hardy, G.H. and Littlewood, J.E., Some problems of 'Partitio numerorum'; (V)., Proc. London Math. Soc. (2) 22 (1923), 46-56.

[5] Huxley, M. N., Large values of Dirichlet polynomials III, Acta Arith. 23 (1975), $435-444$.

[6] Iwaniec, H., A new form of the error term in the linear sieve, Acta Arith. 37 (1980), 307-320.

[7] Iwaniec, H. and Jutila, M., Primes in short intervals, Ark. Mat. 17 (1979), 167-178.

[8] Laborde, M., Nombres presque-premiers dans de petits intervalles, Séminaire de Théorie des Nombres, Bordeaux I, Année 1977-78, exposé $n^{\circ} 15$.

[9] Lou, S.-t. and Yao, Q., The exceptional set of Goldbach numbers in a short interval, Acta Math. Sinica 24 (1981), 269-282.

[10] Mikawa, H., On prime twins, Tsukuba J. Math. 15 (1991), 19-29.

[11] Mikawa, H., On the Brun-Titchmarsh theorem, Tsukuba J. Math. 15 (1991), 31-40.

[12] Montgomery, H.L., Topics in multiplicative number theory, Springer, L. N. Math. $227,1971$.

[13] Montgomery, H.L. and Vaughan, R.C., The exceptional set in Goldbach's problem, Acta Arith. 27 (1975), 353-370.

[14] Motohashi, Y., Sieve methods and prime number theory, Tata Inst. Fund. Res., Lect. Math. Phys. 72, 1983.

[15] Pracher, K., Primzahlverteilung, Springer, 1957.

[16] Ramachandra, K., On the number of Goldbach numbers in small intervals, J. Indian Math. Soc. 37 (1973), 157-170.

[17] Vaughan, R.C., Sommes trigonométriques sur les nombles premiers, C.R. Acad. Sci. Paris Sér. A 286 (1977), 981-983.

[18] Vinogradov, I. M., Representation of an odd number as a sum of three primes, Dokl. Akad. Nauk SSSR 4 (1934), 185-187.

[19] Wolke, D., Über das Primzahl-Zwillingsproblem, Math. Ann. 283 (1989), 529-537.

[20] Yao, Q., The exceptional set of Goldbach numbers in a short interval, Acta Math. Sinica 26 (1982), 315-322. 